\title{
Vegetation and Flora of the Sand Deposits of the Mississippi River Valley in Northwestern Illinois
}

J.E. Ebinger, L.R. Phillippe, R.W. Nÿboer, W.E. McClain, D.T. Busemeyer, K.R. Robertson, and G.A. Levin 
Illinois Natural History Survey, David L. Thomas, Chief

A Division of the Illinois Department of Natural Resources

\author{
Illinois Natural History Survey \\ Distribution Office \\ I-Building \\ 1816 South Oak Street \\ Champaign, IL 61820
}

\title{
Citation:
}

Ebinger, J.E., L.R. Phillippe, R.W. Nÿboer, W.E. McClain, D.T. Busemeyer, K.R. Robertson, and G.A. Levin. 2006. Vegetation and flora of the sand deposits of the Mississippi River valley in northwestern Illinois. Illinois Natural History Survey Bulletin 37(6):191-238.

\section{Editor: Charles Warwick}

\section{US ISSN 0073-4918}

Printed by authority of the State of Illinois

P0109796-.75M-09-06

Printed with soy ink on recycled and recyclable paper.

Equal opportunity to participate in programs of the Illinois Department of Natural Resources (IDNR) and those funded by the U.S. Fish and Wildlife Service and other agencies is available to all individuals regardless of race, sex, national origin, disability, age, religion, or other non-merit factors. If you believe you have been discriminated against, contact the funding source's civil rights office and/or the Equal Employment Opportunity Officer, IDNR, One Natural Resources Way, Springfield, IL 62702-1271; 217/785-0067; TTY 217/782-9175. 


\section{Vegetation and Flora of the Sand Deposits of the Mississippi River Valley in Northwestern Illinois}

J.E. Ebinger, L.R. Phillippe, R.W. Nÿboer, W.E. McClain, D.T. Busemeyer, K.R. Robertson, and G.A. Levin 


\section{ACKNOWLEDGMENTS}

The authors thank the Illinois Department of Natural Resources for funding parts of this project through the Illinois Wildlife Preservation Fund as well as other funding sources, the Illinois Nature Preserves Commission for allowing access to the nature preserves, the United States Army for access to the Savanna Army Depot when it was under their control, and the United States Fish and Wildlife Service for access to the Lost Mound Unit of the Upper Mississippi River Wildlife and Fish Refuge in 2004 and 2005. We also thank Dr. Gordon Tucker, Eastern Illinois University, for his help in identification of the Cyperaceae. Special thanks go to Dr. John B. Taft, scientist at the Illinois Natural History Survey, for his help with the statistical analysis, particularly the Principal Components Analysis (PCA). 


\section{Vegetation and Flora of the Sand Deposits of the Mississippi River Valley in Northwestern Illinois}

J.E. Ebinger, L.R. Phillippe, R.W. Nÿboer, W.E. McClain, D.T. Busemeyer, K.R. Robertson, and G.A. Levin

Correspondence to:

J.E. Ebinger

Emertitus Professor of Botany

Eastern Illinois University Charleston, IL 61920 USA jeebinger@ux1.eiu.edu 


\section{CONTENTS}

Acknowledgments......................... ii

Abstract.................................. 191

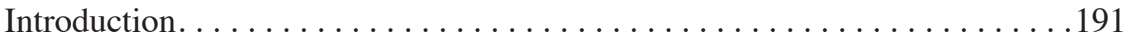

Study Sites . . . . . . . . . . . . . . . . . . . . . . . . . 192

Lost Mound Unit of the Upper Mississippi River

Wildlife and Fish Refuge . . . . . . . . . . . . . . . . . . . . . . 192

Ayers Sand Prairie Nature Preserve . . . . . . . . . . . . . . . . . . . . 194

Thomson-Fulton Sand Prairie Nature Preserve . . . . . . . . . . . . . . 194

Big River State Forest. . . . . . . . . . . . . . . . . . . . . . . . . . . . 194

Materials and Methods. . . . . . . . . . . . . . . . . . . . . . . . . . . . . 195

Vascular Plant Species and Community Types . . . . . . . . . . . . . . 195

Ground Layer Sampling . . . . . . . . . . . . . . . . . . . . . . . . 195

Overstory Sampling . . . . . . . . . . . . . . . . . . . . . 195

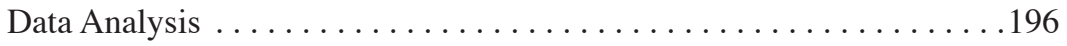

Results. . . . . . . . . . . . . . . . . . . . . . . . . . . . . . . 197

Lost Mound. . . . . . . . . . . . . . . . . . . . . . . . . . . . . . . . 197

Ayers Sand Prairie Nature Preserve . . . . . . . . . . . . . . . . . . . . 204

Thompson-Fulton Sand Prairie Nature Preserve . . . . . . . . . . . . . 206

Big River State Forest. . . . . . . . . . . . . . . . . . . . . 206

Data Analysis and Site Similarity . . . . . . . . . . . . . . . . . 207

Discussion. . . . . . . . . . . . . . . . . . . . . . . . . .211

Historical Summary . . . . . . . . . . . . . . . . . . . . . . . . 211

Bunch-Grass Association of Gleason . . . . . . . . . . . . . . . . . . 211

Other Illinois Sand Deposits . . . . . . . . . . . . . . . . . . . . . . 213

Management Implications. . . . . . . . . . . . . . . . . . . 216

Adventive Species. . . . . . . . . . . . . . . . . . . . . . . . . 216

Forest and Savanna Communities. . . . . . . . . . . . . . . . 217

Literature Cited . . . . . . . . . . . . . . . . . . . . . . . . 218

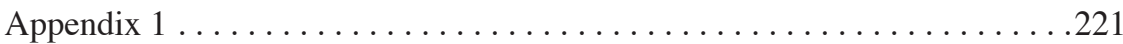




\section{ABSTRACT}

This study was undertaken to determine vascular plant species composition, vegetation structure, and floristic quality of the major plant communities in the windblown sand deposits of northwestern Illinois during the growing seasons of 2002 through 2005. The major plant communities of the Ayers Sand Prairie Nature Preserve in Carroll County, Big River State Forest in Henderson County, Lost Mound Unit of the Upper Mississippi River Wildlife and Fish Refuge in Carroll and Jo Daviess counties, and the Thomson-Fulton Sand Prairie Nature Preserve located in Whiteside County were examined and the importance values determined for the plant species present. Located on broad terraces of the Mississippi River, these nature preserves and natural areas are remnants of a larger grassland/savanna/forest complex that contained extensive marsh; wet, mesic, and dry sand prairie; sand savanna; and sand forest communities. Most of the sand deposits are now cultivated and the original vegetation is found only in protected remnants, some of which are relatively large. The mature dry sand prairies were dominated by Schizachyrium scoparium; other important species were Opuntia macrorhiza, Dichanthelium villosissimum, Ambrosia psilostachya, and Tephrosia virginiana. Other assemblages of prairie and exotic species were encountered in successional sand prairie communities. Generally, the mature prairie communities in these preserves and natural areas had 35 or more species present in the study plots. Savanna and closed canopy forest communities were also examined. The dry sand savannas were dominated by Quercus velutina and $Q$. marilandica, dry sand forests were dominated by $Q$. velutina, and dry-mesic sand forests were dominated by $Q$. alba and $Q$. velutina.

\section{INTRODUCTION}

Glacial outwash, windblown sand deposits are common in the northern half of Illinois due to erosional events associated with Wisconsian glaciation (Willman and Frye 1970, Schwegman 1973, King 1981). The most extensive are the Kankakee sand deposits in northeastern Illinois and the Illinois River sands of Cass, Mason, and Tazewell counties in central Illinois. Numerous smaller sand deposits also occur, including the sands along the upper Mississippi River and its tributaries, the Green River Lowlands sand deposits of Lee and Henry counties in northwestern Illinois, and the Chicago Lake plain and beaches along Lake Michigan in northeastern Illinois.

These sand deposits, named the Parkland Sand or the Parkland Formation, consist of windblown sand in dunes and in sheetlike deposits between and bordering the dunes (Willman and Frye 1970). The dunes are usually found on terraces along the major river valleys in the northern half of Illinois, and consist of medium-grained sands that are sorted by wind from the underlying glacial outwash. These sands were reworked by wind forming the dune and swale topography characteristic of these deposits. Dunes 6 to 12 meters high are common and occasional dunes to $30 \mathrm{~m}$ tall are encountered (Gleason 1910).
Extensive glacial outwash, windblown sand deposits are scattered throughout the lowlands of the Mississippi River in northwestern Illinois (Fig. 1). Referred to as the Mississippi River Section of the Illinois River and Mississippi River Sand Areas Natural Division, these scattered deposits occur from Jo Daviess County south to Henderson County (Schwegman 1973). Some of these deposits were formed when glacial lakes (Lake Milan and Lake Cordova) in Carroll, Henry, Rock Island, and Whiteside counties drained (Fig. 1). Others were deposited during flood events during the retreat of the Wisconsin Glacier when moraines and ice dams were breached and glacial lakes to the north of Illinois drained (Willman and Frye 1970).

Dry habitats are characteristic of sand deposits, and the commonly associated species are those adapted to xeric conditions. However, plant communities of sand deposits are extremely diverse and include sand ponds (McClain et al. 1997), marshes and sedge meadows (Handel et al. 2003, Feist et al. 2006), prairies (Handel et al. 2003, McClain et al. 2003, 2004, Phillippe et al. 2004), savannas and woodlands (McDowell et al. 1983, Johnson and Ebinger 1992, 1995), closed forests (Jenkins et al. 1991, Coates et al. 1992, McClain et al. 2002), and flatwoods (McDowell et al. 1983). 
Some of the most comprehensive early work completed on the vegetation of Illinois sand deposits was undertaken in the early 1900 s by Dr. Henry Allen Gleason, an ecologist and plant geographer then at the Illinois Natural History Survey, and by Arthur G. Vestal, a botanist at the University of Illinois (Hart and Gleason 1907, Gleason 1910, Vestal 1913). These authors described the principal plant communities and discussed the animals associated with these sand deposits, particularly the insects.

Except for the early work by Gleason (1910), little has been published concerning the vegetation of sand deposits along the Upper Mississippi River valley in northwestern Illinois. Though most of these scattered sand deposits are now under cultivation, a fairly extensive preserve system has maintained some of this former diversity. The present study was undertaken to determine vascular plant species composition, vegetation structure of the different plant communities based on the life forms of the species present, and the floristic quality of the major plant communities of the nature preserves and other natural areas located in the windblown sand deposits of the Mississippi River Section of the Illinois River and Mississippi River Sand Areas Natural Division in northwestern Illinois.

\section{STUDY SITES}

All of the study sites are located within $150 \mathrm{~km}$ of each other, and are within a few $\mathrm{km}$ of the Mississippi River (Fig. 1). The climate associated with these sand deposits is continental with warm summers and cold winters. Based on weather data from Dixon, Illinois, $50 \mathrm{~km}$ east of the Mississippi River near the middle of the study area, mean annual precipitation is $94.7 \mathrm{~cm}$, with June having the highest rainfall $(12.4 \mathrm{~cm})$. Mean annual temperature is $8.5^{\circ} \mathrm{C}$ with the hottest month being July (average of $22.3^{\circ} \mathrm{C}$ ), and the coldest January (average of $-7.9^{\circ} \mathrm{C}$ ). The average number of frost-free days is 161 (Midwestern Regional Climate Center 2005).

\section{Lost Mound Unit of the Upper Mississippi River Wildlife and Fish Refuge: Lost Mound is located in northwest Carroll and southwest Jo Daviess counties on the former Savanna Army Depot $\left(42.2410^{\circ} \mathrm{N},-90.3380^{\circ} \mathrm{W}\right.$ [WGS84/}

NAD83]). Gleason (1910), in his classic monograph "Vegetation of the Inland Sand Deposits of Illinois," first described this extensive prairie, which was known as "The Prairie" by local residents. Little of the area was destroyed by cultivation, as grazing was the primary agricultural use of the area. In 1918 the U.S. army purchased most of "The Prairie" to use as an artillery test range. While ownership by the army prevented the large-scale conversion of this area to row crops, the landscape was damaged with the construction of warehouses and other structures that were used to store munitions, and the roads and railroads used to transport them.

The army's mission required preventing wildfires in the extensive remaining prairie. The army restricted the potential for fires by introducing grazing to the area beginning in the late 1940s. Whereas cattle were the primary means of reducing vegetation cover, sheep were used for a time in the early 1950s. Recent cattle grazing leases typically began in late March and extended until November, with 1,000 to 1,200 cattle reducing the vegetation to a lawnlike condition (Robertson et al. 1997). Areas where watering tanks were established had even greater disturbance to the surrounding vegetation, often with extensive areas of bare sand exposed. Where cattle had access to the Mississippi River, side slopes of the sand bluffs were rutted and eroded. Also, changes in the river's hydrology to maintain the navigational channel, have created higher water levels that caused additional bluff erosion and sloughing. Cattle helped maintain some of the blowout communities by increasing disturbance. Recent grazing leases also had provisions to improve the grasslands. Juniperus virginiana (red cedar) and other brush were removed, and some herbaceous vegetation was planted. As part of this "improvement" program, some areas were seeded with a no-till drill to Eurasian cool-season grasses and adventive legumes, particularly Bromus inermis (awnless brome grass) and Trifolium arvense (rabbit-foot clover).

Even with the disturbances, the Illinois Natural Areas Inventory recognized most of the Savanna Army Depot as a statewide significant natural area because of the size of the prairie remnant, the potential for recovery, and the many rare plants and animals present (White 1978). Bowles and Jones (1995) noted the locations of numerous state-listed plants and 


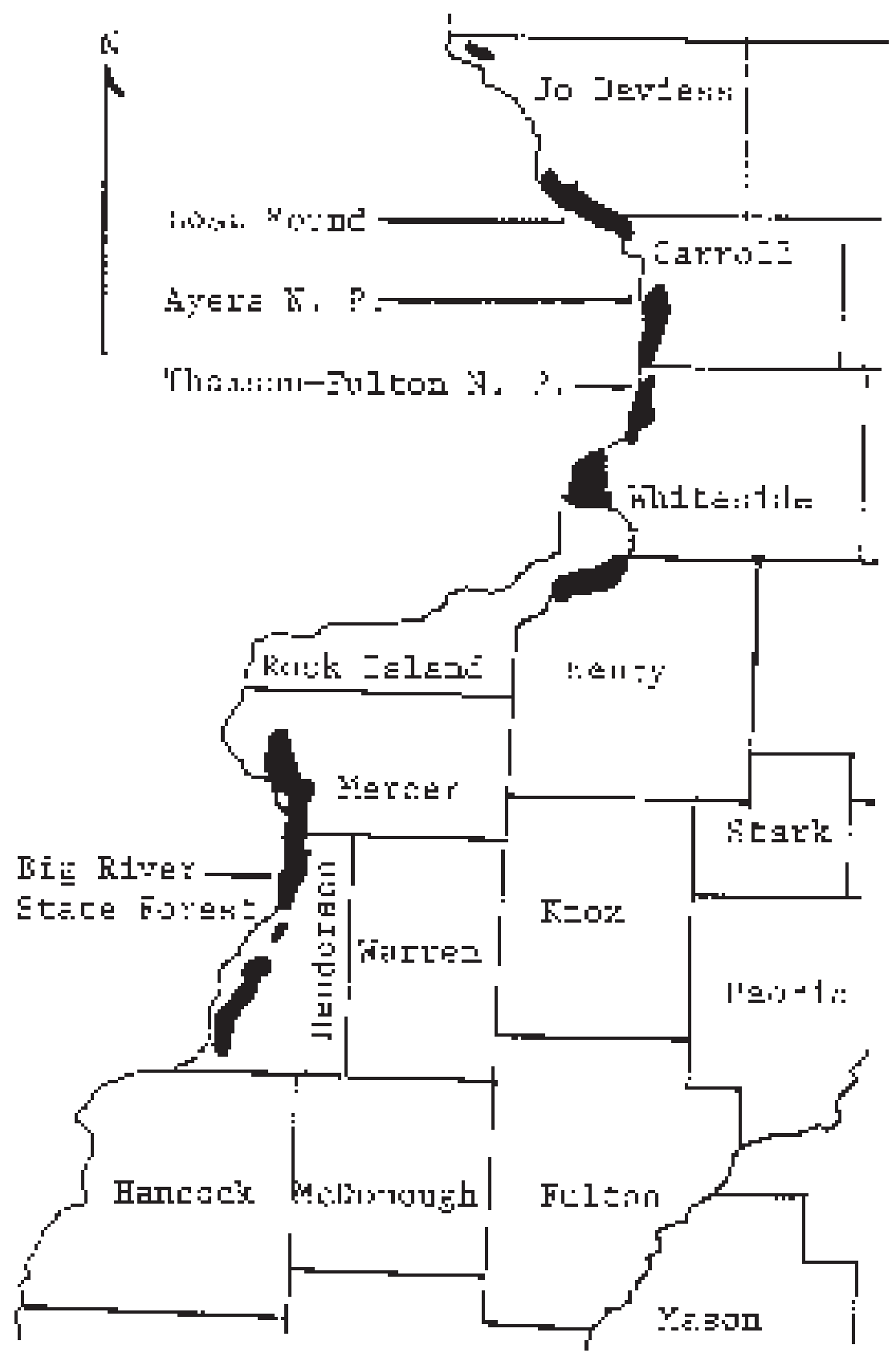

Figure 1. Distribution of sand deposits along the Mississippi River in northwestern Illinois from Jo Daviess County to Henderson County, Illinois. The general location of the four natural areas and nature preserves studies are also included. 
prairies of high natural quality on the depot. As a result, staff of the Illinois Department of Natural Resources persuaded the army to fence some areas to exclude cattle, reduce the grazing period, and decrease the number of cattle on the depot. Grazing ceased in the late 1990s as the military mission was ended at the depot. The land first was transferred to the U.S. Fish and Wildlife Service in 2003. Some of the prairie parcels sampled have been transferred to the Illinois Department of Natural Resources and the Jo-Carroll Local Redevelopment Authority in later years.

The major soil type of Lost Mound is Sparta loamy sand that developed under prairie vegetation. This soil is found on flat to sloping areas, is excessively drained, and consists of deep, dark brown, friable, coarse sand that is underlain by fine loose yellow sand that is often exposed in blowouts (Tegeler 1996). The savanna soils are mostly on steep slopes and are classified as Chelsea loamy sands, which are excessively drained, dark grayish brown in color, and relatively thin; while the nearly level upland forest soils are classified as Bloomfield loamy fine sand and have a similar structure. The floodplain forest soils at Lost Mound are Birds silt loam, which are nearly level, poorly drained, and dark gray-brown in color.

\section{Ayers Sand Prairie Nature Preserve: This} preserve is located in northwestern Carroll County about $3 \mathrm{~km}$ south of Savanna (SE1/4 S24 T24N R3E; $42.0535^{\circ} \mathrm{N},-90.1051^{\circ} \mathrm{W}$ [WGS84/NAD83]). This 46-ha area was dedicated as an Illinois Nature Preserve in 1974. Since dedication the preserve has been recovering from past grazing, off-road vehicle use, cultivation, and other disturbances. The southeastern and northwestern thirds of the preserve are recovering from heavy grazing and cultivation. Parts of the cultivated areas are rapidly reverting to sand prairie vegetation, while Bromus inermis and other cool-season introduced grasses dominate an extensive area in the southeastern part of the preserve. Some blowouts are present in the preserve, though most are now revegetated. The southwestern third of the preserve contains some high-quality dry sand prairie. This area is probably still recovering from past grazing, but was not cultivated. The Illinois Natural Area Inventory considered most of the preserve to be "Grade C" dry sand prairie due to extensive disturbances, though parts of the southwestern section were listed as "Grade B" with a few small areas of "Grade A" (White 1978). The soils of the preserve are Sparta loamy sands (Ray et al. 1975).

\section{Thomson-Fulton Sand Prairie Nature}

Preserve: This preserve is located in extreme northwestern Whiteside County about $6 \mathrm{~km}$ northeast of Fulton, Illinois (SW1/4 S1 and SE1/4 S2 T22N R3E; $41.9253^{\circ} \mathrm{N}$, $-90.1113^{\circ} \mathrm{W}$ [WGS84/NAD83]), immediately south of the Carroll/Whiteside County line. It is a small part of the Thomson Sand Area that extends north into Carroll County. In Whiteside County this sand deposit covers nearly $85 \mathrm{~km}^{2}$ (Smith et al. 1928). The preserve contains about 15 ha of sand prairie that are currently recovering from past grazing, off-road vehicle use, cultivation, and other disturbances. In portions of both the southern and northern parts of this prairie, several ha were plowed and planted to watermelons the year before being dedicated as a preserve by the Illinois Department of Conservation in 1970. This cultivated area is reverting to sand prairie vegetation. Disturbance by off-road vehicles increased the size and number of blowouts in the preserve. These areas are now recovering, many being revegetated, but some contain moving sand. Also, pines that were planted in parts of the prairie prior to acquisition, have been mostly removed. The Illinois Natural Area Inventory considered the area to be mostly "Grade C" dry sand prairie due to extensive disturbances (White 1978). The soils of the preserve are mostly Sparta loamy sand (Sabata 1995).

Big River State Forest: This state forest, which contains a few natural areas, is located in northwestern Henderson County, about $8 \mathrm{~km}$ north of Oquawka (S24, S25, S36 T12N R5W; $40.9920^{\circ} \mathrm{N},-90.9205^{\circ} \mathrm{W}$ [WGS84/NAD83]). Two natural areas within the state forest were studied; a dry sand prairie (NW1/4 S36) and a degraded dry sand savanna (SW1/4 S25). Both sites have been subjected to past disturbances. The dry sand prairie was heavily grazed in the past and pines were planted along the east edge. The dry sand savanna was probably clear-cut soon after settlement and has been subjected to more recent cutting and fire suppression. The Illinois Natural Area Inventory considered most of the dry sand prairie to be of "Grade B" quality, while the dry sand savanna was listed as 
"Grade C" due to the young trees of small size, fire suppression, and other disturbances (White 1978). The soils of the natural areas studied are light colored Plainfield sand and medium-dark colored Oquawka sand that are water-deposited sands of the Mississippi River terrace that have been reworked by wind (Veale and Wascher 1956).

\section{MATERIALS AND METHODS}

\section{Vascular Plant Species and Community}

Types: The natural areas studied were visited a minimum of five times each year throughout the growing seasons of 2002 through 2005, except for the Lost Mound Unit, which was extensively studied on numerous earlier trips during the growing seasons of 1996 and 1997 by some of the authors (Robertson et al. 1997). Voucher specimens of each plant species were collected, identified, and deposited in the herbarium of the Illinois Natural History Survey, Champaign, Illinois (ILLS), and the Stover-Ebinger Herbarium of Eastern Illinois University, Charleston, Illinois (EIU). The species encountered are listed in Appendix I. This list of taxa includes the citation of voucher specimens of nearly all species that have been found in the natural areas studied, as well as a few taxa that were observed but not collected. The list also includes a few species reported by the Illinois Natural Area Inventory (INAI) for which vouchers could not be located. Criteria for designating adventive (non-native) species followed Mohlenbrock (2002), Gleason and Cronquist (1991), and Taft et al. (1997). Nomenclature follows Mohlenbrock (2002). We recorded the location of threatened and endangered plant species listed by Herkert and Ebinger (2002).

The plant communities encountered were described, for the most part, using the classification system of White and Madany (1978). All of the sand prairie communities examined during the present study would be described as various successional stages of a dry sand prairie, including the blowout and blowing sand communities discussed below. In some instances we added modifiers in parenthesis to indicate successional trends, and sometimes the dominant species when discussing a particular community. We consider a mature-to-late successional sand prairie to be equivalent to Grades A and B used by the Illinois Natural Area Inventory (INAI), while successional sand prairie to be equivalent to a low Grade B or C (White 1978). The INAI grading criteria are based on the perceived successional state of the vegetation with Grade A (essentially not degraded \{high floriistic quality]) to Grade $\mathrm{E}$ (highly disturbed [i.e., cropland]).

Ground Layer Sampling: In the late summers of 2004 and 2005 transects were located randomly along cardinal compass directions within the sand prairie communities studied. These transects were located using aerial photographs and ground observation to ensure that they did not cross community boundaries. Within each community a 50-m-long transect was located. Along each transect, $1-\mathrm{m}^{2}$ quadrats were alternately located at $1-\mathrm{m}$ intervals ( $n=50 /$ transect). A random numbers table was used to determine the number of meters (0 to 9) a quadrat was located from the transect line. In some areas (Ayers Sand Prairie Nature Preserve, Thomson-Fulton Sand Prairie Nature Preserve, Big River State Forest) more than one 50-m transect was completed for each habitat type. Only the first transect completed in each habitat type was used in the calculations in this paper. Species cover was determined using the Daubenmire (1959) Cover Class System as modified by Bailey and Poulton (1968). The modified Daubenmire cover scale is as follows: Class 1 $=0$ to $1 \%$; Class $2=>1$ to $5 \%$; Class $3=>5$ to $25 \%$; Class $4=>25$ to $50 \%$; Class $5=>50$ to $75 \%$; Class $6=>75$ to $95 \%$; Class $7=>95$ to $100 \%$. Importance Value (IV) was determined by summing relative cover and relative frequency (total possible $=200$ ).

Overstory Sampling: Savanna and forest communities at Lost Mound and Big River were studied in the late summer of 2005. These areas were surveyed by dividing a portion of each savanna or forest community into contiguous quadrats $25 \mathrm{~m}$ on a side. These sample quadrats were located near the central part of each study area and more than $50 \mathrm{~m}$ from the nearest woodland edge. All living and dead-standing woody individuals $\geq 10.0$ $\mathrm{cm}$ dbh were identified and their diameters recorded. From these data, living-stem density (stems/ha), basal area $\left(\mathrm{m}^{2} / \mathrm{ha}\right)$, relative density, relative dominance, importance value (IV), and average diameter $(\mathrm{cm})$ were calculated for each species. Determination of the IV follows the procedure used by McIntosh (1957), and is the sum of the relative density and relative 
dominance (basal area) for a total sum of 200. Dead-standing density (stem/ha) and basal area $\left(\mathrm{m}^{2} / \mathrm{ha}\right)$ were also determined. Woody understory composition and density (stems/ha) were determined using nested circular plots 0.0001 , 0.001 , and 0.01 ha in size located at $15-\mathrm{m}$ intervals along randomly located east-west transects within each study area. Four additional 0.0001ha circular plots were located $6 \mathrm{~m}$ from the center points of each plot center along cardinal compass directions. In the 0.0001-ha plots, woody seedlings ( $\leq 50 \mathrm{~cm}$ tall) were counted; in the 0.001 -ha circular plots small saplings $(>50$ $\mathrm{cm}$ tall and $<2.5 \mathrm{~cm} \mathrm{dbh}$ ) were recorded; and in the 0.01-ha circular plots large saplings (2.5-9.9 $\mathrm{cm} \mathrm{dbh}$ ) were tallied.

Data Analysis: The Floristic Quality Index (FQI) was determined for each nature preserve and natural area using the coefficient of conservatism (CC) assigned each species based on a species tolerance to disturbance and its fidelity to habitat integrity (Taft et al. 1997). The FQI, therefore, is a weighted index of species richness $(\mathrm{N}=$ number of species present on a site), and is the arithmetic product of the average coefficient of conservatism $(\mathrm{C}$-Value $=$ the average of all species CCs) multiplied by the square root of the native species richness $(\sqrt{ } \mathrm{N})$ of an inventory site: $\mathrm{FQI}=\mathrm{C}$-Value $(\sqrt{\mathrm{N}})$. For relatively small areas that are intensively studied, the FQI gives a rapid means of comparison and an indication of the floristic integrity of the site. Using the FQI along with other floristic measures, such as quadrat-based sampling methods, provides a meaningful way of making comparisons among sites. Prairies with an FQI of 35 or higher are usually considered goodquality natural areas (Taft et al. 1997). Though area dependent, the FQI can still be useful in explaining the variation among sites of similar size and habitat (Taft et al. 2006). In our study, the FQI was determined for each of the four natural areas studied, as well as for each of the 15 sand prairie communities surveyed.

The Sorensen Index of Similarity (ISs) was used to determine the degree of vegetation similarity between the prairie areas surveyed throughout the Mississippi River sand deposits (Mueller-Dombois and Ellenberg 1974). In this index [ISs $=2 \mathrm{C} / \mathrm{A}+\mathrm{B} \times 100]$, A equals the number of species in the first community, $B$ equals the number of species in the second community, and $\mathrm{C}$ equals the number of species common between the two communities.

Cluster analysis was used to produce a hierarchical classification of sample transects from the sand prairie study sites (PC-ORD; McCune and Mefford 1999) and a variety of distance measures and linkage methods were explored. While there was some variation in the results among methods, cluster analysis using the Euclidean (Pythagorean) distance measure and Ward's linkage method produced a dendrogram similar to Sorensen Distance Measure and Farthest Neighbor Linkage method. This consensus of group clusters was integrated into ordination biplots using both Detrended Correspondence Analysis (DCA) and Principal Components Analysis (PCA). Since all samples (transects) were from a similar vegetation type (dry to dry-mesic sand prairie) and included many shared species, the dataset was amenable to analysis using the linear response model in PCA. Gradient lengths on the first DCA axis (2.5 standard deviations [SD]) were within the range where both linear and Gaussian methods can be effective ordination techniques (Ter Braak and Prentice 1988, Ter Braak 1995). Most plots (i.e., transects [12 of 15]) fall within 2 SD on the first DCA axis indicating most species are responding with little variation over the observed range of environmental conditions. Under these circumstances, a linear response model (e.g., PCA) is appropriate. The graphical depiction of the PCA biplot also was more readily interpretable compared to DCA; consequently, PCA was the preferred ordination technique with this dataset. A correlation matrix was used for the ordination with species scores divided by their standard deviation. The top-ranking 75 species based on importance values were used for the ordination; the remaining 45 species in the dataset all were scarce (present in only one or two transects) and occurred in low percentage cover.

Constrained ordination using communitylevel parameters as environmental variables (i.e., native species richness, adventive species richness, species density [average species number per quadrat], percent bare ground, mean coefficient of conservatism) with Redundancy Analysis explained $91 \%$ of the species-environment relations. However, percent bare ground was the only variable explaining a significant amount of the variation $(P=0.01)$. 


\section{RESULTS}

\section{Lost Mound}

A total of 621 species in 353 genera and 108 families was documented (Appendix I). Ferns, fern-allies, and gymnosperms accounted for 21 species in 11 families and 15 genera, while 157 were monocots in 18 families and 75 genera, and 443 were dicots in 79 families and 263 genera. Adventive (exotic) species accounted for 136 taxa, about $22 \%$ of all species. Five state-threatened species (Herkert and Ebinger 2002) were recorded: Besseya bullii (kitten tails), Cyperus grayoides (sand prairie flatsedge), Elymus trachycaulus (bearded wheat grass), Equisteum pratense (meadow horsetail), and Salvia azurea (blue sage); and seven stateendangered species were encountered: Bouteloua gracilis (blue grama), Ceanothus herbaceus (redroot), Hudsonia tomentosa (beach heather), Mirabilis hirsuta (hairy umbrellawort), Opuntia fragilis (fragile prickly pear), Orobanche fasciculata (clustered broomrape), and Polanisia jamesii (James' clammyweed). The FQI was determined only for the dry sand prairie communities at Lost Mound because the FQI is meaningful for only small areas. The FQI for sand prairie communities at this site when adventive species were included was 59.70 with a mean C-value of 2.97 , and with the adventive species excluded from the calculations the FQI was 80.00 with a mean C-value of 3.98 .

\section{Blowout Community (early successional} dry sand prairie): Blowouts were numerous at Lost Mound. All had a sparse vegetation cover with relatively few species. In the community surveyed, seven species dominated, all with high mean covers and IVs. Five of these species, Carex muhlenbergii (Muhlenberg's sedge), Dichanthelium villosissimum (hairy panic grass), Aristida tuberculosa (needle grass), Cyperus schweinitzii (Schweinitiz' sedge), and Panicum virgatum (switch grass) were the native graminoid taxa; whereas Croton glandulosus (sand croton) and Ambrosia psilostachya (western ragweed) were the dominant forbs (Table 1). The 16 remaining species were mostly native dry sand prairie components that were common in surrounding plant communities. The only adventive species, Mollugo verticillata (carpetweed) was uncommon with an IV of 0.8. Bare ground and litter had a mean cover of $63 \%$. This community is the Blowout Formation of Gleason (1910), who describes the four major associations of this formation (windward slope, basin, blowsand, and deposition), and discusses the stages of succession to the bunch-grass association.

\section{Blowing Sand Community (early succes- sional dry sand prairie): Areas of blowing} sand, generally associated with dune ridges, were common at Lost Mound. These open areas were the result of past disturbances, particularly grazing (Table 1). On the dune ridge Hudsonia tomentosa formed extensive low mounds and dominated with a mean cover of $20.4 \%$ and an IV of 41.0. Tephrosia virginiana (goat'srue), Dichanthelium villosissimum, Ambrosia psilostachya, and Andropogon gerardii (big bluestem) followed in IV. Most of the other species found in the plots were common sand prairie species. The adventive Rumex acetosella (sour dock) was common, ranking eighth in $\mathrm{IV}$, and found in about $50 \%$ of the plots. The adventive cool-season Poa pratensis (Kentucky blue grass) was present but infrequent. Bare ground and litter had a mean cover of $41 \%$. This community is the Hudsonia Association of Gleason (1910), which he commonly found in the Hanover region (Lost Mound).

\section{Dry Sand Prairie Community (successional with cool season grasses common): Much} of Lost Mound had been subjected to cattle grazing, which kept the vegetation cover sparse and low, and helped decrease the frequency and intensity of "wild" fires. Many of these areas were seeded in cool-season, Eurasian grasses, particularly Poa pratensis and Bromus inermis. Generally this was done with minimal or no ground preparation. The resulting pastures had a relatively high importance of the cool season grasses, along with a fairly well-developed sand prairie community with most of the prairie grasses and forbs still present (Table 2). In the three areas surveyed, Poa pratensis was second in importance on two sites and sixth in importance on the third. On this third site (Primms Prairie), Bromus inermis was fifth in importance and the adventive Rumex acetosella (sour dock) was second. On all three sites native prairie grasses and forbs were common, with Poa pratensis, Bromus inermis, and Rumex acetosella the chief adventive species encoun- 
Table 1. Frequency (\%), mean cover (\% of total area) and importance value (I.V.) of the ground layer species encountered in the fall 2005 surveys of a blowout community and a blowing sand community at Lost Mound, Jo Daviess County, Illinois. (* non-native species)

\begin{tabular}{|c|c|c|c|c|c|c|}
\hline \multirow{2}{*}{ Species } & \multicolumn{3}{|c|}{$\begin{array}{c}\text { Blowout Community } \\
\text { Area } 1(n=50)\end{array}$} & \multicolumn{3}{|c|}{$\begin{array}{c}\text { Blowing Sand Community } \\
\text { Area } 2(\mathrm{n}=50)\end{array}$} \\
\hline & Freq. $\%$ & $\begin{array}{l}\text { Mean } \\
\text { Cover }\end{array}$ & I.V. & Freq. $\%$ & $\begin{array}{l}\text { Mean } \\
\text { Cover }\end{array}$ & I.V. \\
\hline Carex muhlenbergii & 58 & 5.96 & 25.7 & 48 & 0.59 & \\
\hline $\begin{array}{l}\text { Dichanthellum villosissimum } \\
\text { Aristida tuberculosa. }\end{array}$ & $\begin{array}{l}66 \\
94\end{array}$ & $\begin{array}{l}3.89 \\
3.89\end{array}$ & 24.4 & $\begin{array}{l}66 \\
72\end{array}$ & $\begin{array}{l}3.6 / \\
1.49\end{array}$ & 11.3 \\
\hline Cyperus schweinitzii & 94 & 3.51 & 23.3 & 54 & 0.72 & 8.2 \\
\hline Ambrosia psilostachya & 78 & 2.045 & 19.0 & 70 & 2.74 & 13.1 \\
\hline $\begin{array}{l}\text { Panicum virgatum } \\
\text { Cyperus lupulinus }\end{array}$ & $\begin{array}{l}54 \\
60\end{array}$ & $\begin{array}{l}3.63 \\
0.85\end{array}$ & $\begin{array}{l}18.3 \\
10.8\end{array}$ & $\begin{array}{l}22 \\
40\end{array}$ & $\begin{array}{l}0.94 \\
0.55\end{array}$ & $\begin{array}{l}4.2 \\
5.9\end{array}$ \\
\hline $\begin{array}{l}\text { Tephrosia virginiana } \\
\text { Oenothera clelandii }\end{array}$ & 12 & 2.70 & 9.7 & 36 & 10.92 & 21.9 \\
\hline Paspalum bushii & 12 & 1.03 & 4.8 & ${ }^{4}$ & 0.06 & 0.3 \\
\hline Polygonella articulata & 16 & 0.42 & 3.4 & $3 \overline{2}$ & 0.60 & 4.9 \\
\hline $\begin{array}{l}\text { Chamaesyce geyerl } \\
\text { Diodia teres }\end{array}$ & $\begin{array}{r}16 \\
6\end{array}$ & 0.13 & $\begin{array}{l}3.0 \\
1.2\end{array}$ & 2 & 0.01 & 0.2 \\
\hline Leptoloma cognatum & 2 & 0.30 & 1.2 & 5 & & \\
\hline $\begin{array}{l}\text { Carex tonsa } \\
\text { Koeleria macrantha }\end{array}$ & 6 & 0.08 & 1.0 & 54 & 2.08 & 10.3 \\
\hline * Mollugo verticillata & 4 & 0.07 & 0.8 & -- & & \\
\hline Conyza canadensis & 4 & 0.02 & 0.7 & -- & -- & - \\
\hline $\begin{array}{l}\text { Triplasis purpurea } \\
\text { Asclepias viridiflora }\end{array}$ & ${ }^{4}$ & 0.01 & 0.3 & --- & -- & - \\
\hline Monarda punctata & 2 & 0.01 & 0.3 & -- & -- & - \\
\hline $\begin{array}{l}\text { Sporobolus cryptandrus } \\
\text { Hudsonia tomentosa }\end{array}$ & 2 & 0.01 & 0.3 & 68 & 2039 & 410 \\
\hline Andropogon gerardii & -- & -- & -- & 36 & 4.31 & 11.4 \\
\hline * Rumex acetosella & -- & -- & - & 50 & 1.62 & 8.8 \\
\hline Cyperus grayoides & -- & -- & -. & 24 & 0.61 & 4.0 \\
\hline Rhus aromatica & -- & -- & -- & 6 & 2.06 & 4.0 \\
\hline $\begin{array}{l}\text { Schizachyrium scoparium } \\
\text { Euphorbia corollata }\end{array}$ & $=-$ & -- & -- & 12 & $\begin{array}{l}1.41 \\
0.79\end{array}$ & 2.8 \\
\hline Lespedeza capitata & -- & -- & -- & 14 & 0.65 & 2.7 \\
\hline $\begin{array}{l}\text { Pollaago nemoralls } \\
\text { *Pog pratensis }\end{array}$ & - & & & $\frac{4}{6}$ & 0.08 & 0.8 \\
\hline Opuntia macrorhiza & - & -- & - & 2 & 0.30 & 0.7 \\
\hline $\begin{array}{l}\text { Plantago patagonica } \\
\text { Brickelia }\end{array}$ & $\ldots$ & & -- & ${ }^{4}$ & 0.02 & 0.5 \\
\hline Lithospermum croceum & - & & - & 2 & 0.06 & 0.3 \\
\hline Asclepias verticillata & -- & -- & -- & 2 & 0.01 & 0.2 \\
\hline $\begin{array}{l}\text { Aster sericeus } \\
\text { Bouteloua hirsuta }\end{array}$ & $\begin{array}{l}-- \\
--\end{array}$ & & & 2 & 0.01 & 0.2 \\
\hline Sporobolus clandestinus & -- & & 2000 & & 0.01 & 200. \\
\hline Bare ground and litter & & & & & 40.60 & \\
\hline
\end{tabular}


tered (Table 2). On these three sites the mean cover of bare ground and litter ranged from 13 to $30 \%$.

\section{Dry Sand Prairies Community (mid-succes-} sional): On interdunal areas and lower dune slopes, mid-successional dry sand prairies dominated by Sporobolus clandestinus (dropseed) and Selaginella rupestris (rock spikemoss) were common. These two species combined accounted for over one-third of the importance value. Sporobolus cryptandrus (sand dropseed) and $S$. compositus were also present, but in lower numbers. Other common graminoids included Koeleria macrantha (June grass), Leptoloma cognitum (fall witch grass), Cyperus lupulinus (flatsedge), and C. schweinitzii; the common forbs were Ambrosia psilostachya, Asclepias verticillata (horsetail milkweed), and Opuntia macrorhiza (plains prickly pear) (Table 3).

On upper dune slopes and dune ridges, another mid-successional dry sand prairie community was sometimes found. Heterotheca spartea (porcupine grass), Opuntia macrorhiza, and Selaginella rupestris dominated the community, and along with the subdominants Schizachyrium scoparium (little bluestem) and Ambrosia psilostachya, accounted for nearly $60 \%$ of the total IV (Table 3 ). In both of these communities few adventive species other than Poa pratensis were encountered. Both communities were heavily grazed in the past and both had a mean cover of bare ground and litter of 22 to $23 \%$.

\section{Dry Sand Prairie Community (mature or} late successional): Excessive grazing and the introduction of cool season grasses and other exotic species have degraded most of the dry sand prairie community at Lost Mound. Some areas, however, have been fenced and have not recently been subjected to heavy grazing. One area, located on a dune ridge and east-facing dune slope, was fenced in 1995 to exclude grazing. During the spring of 2005 a "wildfire" burned the east-facing slope. This burned area is presently dominated by two native species, Ambrosia psilostachya and Schizachyrium scoparium, and the adventive Rumex acetosella (Table 4). Other common species include the prairie forb Aster ericoides (heath aster); two native bunch-grasses, Koeleria macrantha and Leptoloma cognatum; and the native prairie shrub Amorpha canescens (leadplant). These seven species accounted for more than $50 \%$ of the IV. On the unburned dune ridge the dry sand prairie was dominated by Schizachyrium scoparium with an IV of 40.4. Selaginella rupestris, which was second in IV, formed extensive colonies on the surface of the sand between the other species, while Ambrosia psilostachya ranked third with an IV of 20.1. On this prairie the adventive Rumex acetosella and Potentilla recta (sulfur cinquefoil) ranked fourth and fifth in IV, while native graminoid taxa accounted for the next five species in IV (Table 4). Bare ground and litter had a mean cover of $27 \%$ on the unburned, and $38 \%$ on the burned part of this prairie. This community is the Mixed Consocies of the Bunch-Grass Association described by Gleason (1910).

\section{Dry Sand Savanna Community: Fire sup-} pression, grazing, and other disturbances degraded most of the dry sand savannas at Lost Mound. The savanna surveyed, which had a tree canopy cover of less than $40 \%$, was located in and along the margin of a large stabilized blowout. In parts of this savanna the trees were widely scattered, other areas had nearly $80 \%$ closed canopy. Quercus velutina (black oak) was the only species present that exceeded 10 $\mathrm{cm} \mathrm{dbh}$. This species dominated the seedling and sapling layer and averaged 240 stems/ha ( $\leq 10 \mathrm{~cm} \mathrm{dbh}$.) and $14.323 \mathrm{~m}^{2} /$ ha of basal area (Table 5). Wind action had exposed the large basal caudex of many of the older black oaks showing that these trees probably originated as grubs. Black oak and species of Rubus (dewberries, blackberries, and raspberries), Rhus (sumac), and Prunus (cherries) were common components of the seedling layer. Saplings averaged fewer than 3,000 stems/ha, nearly all less than $2.5 \mathrm{~cm}$ dbh (Table 5).

Dry Sand Forest Community: In the southern third of Lost Mound, at the edge of the Mississippi River, is a large stabilized dune covered by dry sand forest. This forest was dominated by Quercus velutina with 332 stems/ha, 22.959 $\mathrm{m}^{2} /$ ha of basal area, and $95 \%$ of the IV (Table $6)$. The only other species reaching tree size $(\leq 10 \mathrm{~cm} \mathrm{dbh})$ were a few small individuals of Prunus serotina (wild black cherry), Quercus alba (white oak), Fraxinus lanceolata (green ash), and Juglans nigra (black walnut). The seedling and small sapling layers were dense. 
Table 2. Frequency (\%), mean cover (\% of total area), and importance value (I.V.) of the ground layer species encountered in the fall of 2005 in dry sand prairie successional communities dominated by cool season grasses at Lost Mound, Jo Daviess County, Illinois. (*non-native species)

\begin{tabular}{|c|c|c|c|c|c|c|c|c|c|}
\hline \multirow[b]{2}{*}{ Species } & \multicolumn{3}{|c|}{$\begin{array}{l}\text { Schizachyrium/Poa } \\
\text { Community } \\
\text { Area } 3(n=50)\end{array}$} & \multicolumn{3}{|c|}{$\begin{array}{l}\text { Tephrosia/Poa } \\
\text { Community } \\
\text { Area } 4(\mathrm{n}=50)\end{array}$} & \multicolumn{3}{|c|}{$\begin{array}{l}\text { Primms Prairie } \\
\text { Area } 5(n=50)\end{array}$} \\
\hline & Freq. \% & $\begin{array}{l}\text { Mean } \\
\text { Cover }\end{array}$ & I.V. & Freq. \% & $\begin{array}{l}\text { Mean } \\
\text { Cover }\end{array}$ & I.V. & Freq. $\%$ & $\begin{array}{l}\text { Mean } \\
\text { Cover }\end{array}$ & I.V. \\
\hline Schizachyrium scoparium & 100 & 26.58 & 45.6 & 62 & 10.77 & 21.8 & -- & -- & -- \\
\hline *Poa pratensis & 68 & 5.63 & 14.4 & 76 & 17.47 & 32.0 & 88 & 7.18 & 14.3 \\
\hline Ambrosia psilostachya & 72 & 4.98 & 14.1 & 16 & 0.13 & 2.4 & 88 & 12.29 & 20.1 \\
\hline Selaginella rupestris & 46 & 7.02 & 14.1 & 42 & 9.25 & 17.1 & -- & -- & -- \\
\hline Opuntia macrorhiza & 44 & 5.19 & 11.5 & 2 & 0.75 & 1.2 & 4 & 0.02 & 0.3 \\
\hline Koeleria macrantha & 50 & 4.78 & 11.4 & 22 & 1.57 & 4.9 & 28 & 1.36 & 3.4 \\
\hline Cyperus lupulinus & 80 & 1.15 & 9.8 & 20 & 0.20 & 2.9 & 56 & 0.43 & 4.4 \\
\hline Asclepias verticillata & 60 & 1.92 & 8.7 & 28 & 0.73 & 4.8 & 38 & 0.24 & 2.9 \\
\hline Tephrosia virginiana & 24 & 4.68 & 8.7 & 94 & 24.37 & 42.9 & -- & -- & -- \\
\hline *Rumex acetosella & 44 & 0.96 & 5.9 & -- & -- & -- & 94 & 10.22 & 18.1 \\
\hline Dichanthelium villosissimum & 40 & 1.03 & 5.5 & 46 & 2.88 & 10.0 & 50 & 2.55 & 6.4 \\
\hline Helianthus pauciflorus & 28 & 1.31 & 4.6 & -- & -- & -- & -- & -- & -- \\
\hline Leptoloma cognatum & 28 & 1.07 & 4.3 & 10 & 0.49 & 2.0 & 52 & 2.07 & 5.9 \\
\hline Rhus aromatica & 14 & 2.13 & 4.3 & 4 & 2.50 & 3.6 & 14 & 1.78 & 3.0 \\
\hline Carex tonsa & 34 & 0.42 & 4.1 & 12 & 0.11 & 1.7 & -- & -- & -- \\
\hline Aster sericeus & 12 & 1.48 & 3.2 & -- & -- & -- & -- & -- & -- \\
\hline Cyperus schweinitzii & 24 & 0.37 & 3.0 & 2 & 0.01 & 0.3 & 12 & 0.06 & 0.9 \\
\hline Physalis virginiana & 22 & 0.51 & 3.0 & 54 & 2.03 & 10.0 & 30 & 0.55 & 2.7 \\
\hline Polygala polygama & 20 & 0.25 & 2.4 & -- & -- & -- & 8 & 0.04 & 0.6 \\
\hline Plantago patagonica & 20 & 0.15 & 2.3 & 6 & 0.03 & 0.8 & 12 & 0.06 & 0.9 \\
\hline Panicum virgatum & 10 & 0.73 & 2.0 & 14 & 0.12 & 2.0 & 86 & 8.03 & 15.1 \\
\hline Dichanthelium oligosanthes & 14 & 0.32 & 1.9 & -- & -- & -- & 42 & 1.54 & 4.7 \\
\hline Carex muhlenbergii & 14 & 0.22 & 1.8 & 22 & 0.41 & 3.5 & 24 & 0.21 & 2.5 \\
\hline Andropogon gerardii & 2 & 0.75 & 1.2 & -- & -- & -- & -- & -- & -- \\
\hline Conyza canadensis & 10 & 0.15 & 1.2 & -- & -- & -- & 50 & 1.28 & 5.0 \\
\hline Euphorbia corollata & 8 & 0.33 & 1.2 & -- & -- & -- & -- & -- & -- \\
\hline Equisetum laevigatum & 10 & 0.05 & 1.1 & -- & -- & -- & 30 & 0.15 & 2.3 \\
\hline Lithospermum croceum & 6 & 0.32 & 1.0 & -- & -- & -- & 84 & 9.22 & 16.3 \\
\hline Pseudognaphalium obtusifolium & 6 & 0.32 & 1.0 & -- & -- & -- & -- & -- & -- \\
\hline *Achillea millefolium & 8 & 0.04 & 0.9 & -- & -- & -- & 44 & 0.81 & 3.9 \\
\hline Lespedeza capitata & 8 & 0.09 & 0.9 & -- & -- & -- & 44 & 2.41 & 5.7 \\
\hline Dichanthelium perlongum & 6 & 0.18 & 0.8 & 4 & 0.12 & 0.7 & -- & -- & -- \\
\hline Paspalum bushii & 6 & 0.08 & 0.7 & -- & -- & -- & 78 & 5.84 & 12.0 \\
\hline Brickellia eupatorioides & 4 & 0.12 & 0.6 & -- & -- & -- & 52 & 3.23 & 7.3 \\
\hline Erigeron strigosus & 4 & 0.07 & 0.5 & -- & -- & -- & -- & -- & -- \\
\hline Oxalis stricta & 4 & 0.07 & 0.5 & -- & -- & -- & 20 & 0.10 & 1.5 \\
\hline *Kummerowia stipulacea & 4 & 0.02 & 0.4 & -- & -- & -- & -- & -- & -- \\
\hline Polygonит tепие & 4 & 0.02 & 0.4 & -- & -- & -- & -- & -- & -- \\
\hline Callirhoe triangulata & 2 & 0.06 & 0.3 & -- & -- & -- & -- & -- & -- \\
\hline Eragrostis spectabilis & 2 & 0.06 & 0.3 & 2 & 0.06 & 0.4 & 2 & 0.06 & 0.2 \\
\hline Oenothera clelandii & 2 & 0.01 & 0.2 & -- & -- & -- & 4 & 0.07 & 0.4 \\
\hline Sporobolus cryptandrus & 2 & 0.01 & 0.2 & -- & -- & -- & 22 & 0.21 & 1.7 \\
\hline Opuntia fragilis & -- & -- & -- & 88 & 4.44 & 17.8 & -- & -- & -- \\
\hline Solanum carolinense & -- & -- & -- & 28 & 1.75 & 6.1 & 16 & 0.28 & 1.6 \\
\hline Heterostipa spartea & -- & -- & -- & 14 & 0.27 & 2.2 & 8 & 0.09 & 0.7 \\
\hline Viola pedata & -- & -- & -- & 12 & 0.21 & 1.9 & -- & -- & -- \\
\hline Bouteloua hirsuta & -- & -- & -- & 12 & 0.16 & 1.8 & -- & -- & -- \\
\hline Croton glandulosus & -- & -- & -- & 6 & 0.37 & 1.4 & 38 & 0.24 & 2.9 \\
\hline Triplasis purpurea & -- & -- & -- & 4 & 0.12 & 1.2 & -- & -- & -- \\
\hline Tradescantia ohiensis & -- & -- & -- & 6 & 0.08 & 0.9 & -- & -- & -- \\
\hline Sporobolus clandestinus & -- & -- & -- & 4 & 0.02 & 0.6 & 4 & 0.31 & 0.7 \\
\hline Aristida tuberculosa & -- & -- & -- & 2 & 0.06 & 0.4 & -- & -- & -- \\
\hline *Mollugo verticillata & -- & -- & -- & 2 & 0.06 & 0.4 & -- & -- & -- \\
\hline
\end{tabular}




\begin{tabular}{|c|c|c|c|c|c|c|c|c|c|}
\hline \multirow{2}{*}{$\begin{array}{l}\text { Table } 2 \text { cont } \\
\text { Species }\end{array}$} & \multicolumn{3}{|c|}{$\begin{array}{c}\text { Schizachyrium/Poa } \\
\text { Community } \\
\text { Area } 3(n=50) \\
\end{array}$} & \multicolumn{3}{|c|}{$\begin{array}{l}\text { Tephrosia/Poa } \\
\text { Community } \\
\text { Area } 4(n=50)\end{array}$} & \multicolumn{3}{|c|}{$\begin{array}{l}\text { Primms Prairie } \\
\text { Area } 5(n=50)\end{array}$} \\
\hline & Freq. $\%$ & $\begin{array}{l}\text { Mean } \\
\text { Cover }\end{array}$ & I.V. & Freq. $\%$ & $\begin{array}{l}\text { Mean } \\
\text { Cover }\end{array}$ & I.V. & Freq. $\%$ & $\begin{array}{l}\text { Mean } \\
\text { Cover }\end{array}$ & I.V. \\
\hline Chamaescye geyeri & -- & -- & -- & 2 & 0.01 & 0.3 & -- & -- & -- \\
\hline *Bromus inermis & -- & -- & -- & -- & -- & -- & 50 & 9.84 & 14.7 \\
\hline Verbena stricta & -- & -- & -- & -- & -- & -- & 28 & 1.26 & 3.3 \\
\hline Sorghastrum nutans & -- & -- & -- & -- & -- & -- & 14 & 1.83 & 3.1 \\
\hline Monarda punctata & -- & -- & -- & -- & -- & -- & 26 & 0.43 & 2.3 \\
\hline Poinsettia dentata & -- & -- & -- & -- & -- & -- & 18 & 0.14 & 1.4 \\
\hline *Potentilla recta & -- & -- & -- & -- & -- & -- & 14 & 0.27 & 1.3 \\
\hline Strophostyles helvula & -- & -- & -- & -- & -- & -- & 10 & 0.15 & 0.9 \\
\hline Gleditsia triacanthos & -- & -- & -- & -- & -- & -- & 8 & 0.14 & 0.8 \\
\hline Chamaecrista fasciculata & -- & -- & -- & -- & -- & -- & 8 & 0.04 & 0.6 \\
\hline Crotalaria sagittalis & -- & -- & -- & -- & -- & -- & 6 & 0.08 & 0.5 \\
\hline Rosa carolina & -- & -- & -- & -- & -- & -- & 2 & 0.30 & 0.4 \\
\hline *Saponaria officinalis & -- & -- & -- & -- & -- & -- & 4 & 0.07 & 0.4 \\
\hline Senecio plattensis & -- & -- & -- & -- & -- & -- & 4 & 0.12 & 0.4 \\
\hline Physalis heterophylla & -- & -- & -- & -- & -- & -- & 4 & 0.02 & 0.3 \\
\hline Physalis subglabrata & -- & -- & -- & -- & -- & -- & 4 & 0.02 & 0.3 \\
\hline Asclepias syriaca & -- & -- & -- & -- & -- & -- & 2 & 0.06 & 0.2 \\
\hline Juniperus virginiana & -- & -- & -- & -- & -- & -- & 2 & 0.06 & 0.2 \\
\hline Prunus serotina & -- & -- & -- & -- & -- & -- & 2 & 0.06 & 0.2 \\
\hline Cirsium discolor & -- & -- & -- & -- & -- & -- & 2 & 0.01 & 0.1 \\
\hline Phyla lanceolata & -- & -- & -- & -- & -- & -- & 2 & 0.01 & 0.1 \\
\hline *Potentilla argentea & -- & -- & -- & -- & -- & -- & 2 & 0.01 & 0.1 \\
\hline Totals & & 75.63 & 200.0 & & 81.55 & 200.0 & & 87.85 & 200.0 \\
\hline Bare ground and litter & & 30.36 & & & 16.82 & & & 13.08 & \\
\hline
\end{tabular}

Woody seedlings averaged 34,066 stems/ha, small saplings averaged 10,533 stems/ha, but large saplings averaged only 468 stems/ha (Table 6). Rubus allegheniensis (common blackberry) dominated the seedling and small sapling layer with 17,188 and 2,813 stems/ha, respectively. Seedlings and small saplings of Cornus racemosa (gray dogwood) and Prunus virginiana (common chokecherry) were also common (Table 6).

Dry-Mesic Sand Forest Community: Along the northern edge of Lost Mound is a relatively extensive upland sand forest, most of which has been degraded by fire suppression, exotic species invasion, lumbering, and other human activities. Small mature second-growth forest inclusions of a few ha are occasional in this area. Quercus alba and Q. velutina were the dominant species, and together accounted for $69 \%$ of the IV, averaged 177 stems/ha, and had a combined basal area of $23.438 \mathrm{~m}^{2} / \mathrm{ha}$ (Table 7). Twelve other species reached tree size ( $\leq 10 \mathrm{~cm} \mathrm{dbh)} \mathrm{with} \mathrm{Carya} \mathrm{cordiformis}$ (bitternut hickory) and Prunus serotina the most important. Woody seedlings were abundant with 30,158 stems/ha. Quercus alba and Prunus serotina seedlings were the most common, but seedlings of many species of shrubs were also present. Small and large saplings were not abundant, resulting in an open understory (Table 7).

Wet-mesic Floodplain Forest Community: In the floodplain area immediately south of Lock and Dam 12, the hydrology influencing the floodplain forest and backwater sloughs has been altered since the dam was completed in 1939. Extensive wet-mesic floodplain forests grow on the exposed floodplains. Acer saccharinum (silver maple) dominated and accounted for $91 \%$ of the IV (182.9) with 217 stems/ha and a basal area of $34.175 \mathrm{~m}^{2} / \mathrm{ha}$. Small numbers of Ulmus americana (American elm), Fraxinis lanceolata, and Celtis occidentalis (hackberry), were encountered (Table 8). Woody seedlings were common, but few would enter the sapling layer as indicated by the small number of saplings present. 
Table 3. Frequency (\%), mean cover (\% of total area), and importance value (I.V.) of the ground layer species encountered in the fall of 2005 in dry sand prairie mid-successional communities at Lost Mound, Jo Daviess County, Illinois. (*non-native species)

\begin{tabular}{|c|c|c|c|c|c|c|}
\hline \multirow[b]{2}{*}{ Species } & \multicolumn{3}{|c|}{$\begin{array}{c}\text { Sporobolus/Selaginella } \\
\text { Community } \\
\text { Area } 6(\mathrm{n}=50)\end{array}$} & \multicolumn{3}{|c|}{$\begin{array}{c}\text { Heterostipa/Opuntia } \\
\text { Community } \\
\text { Area } 7(n=50)\end{array}$} \\
\hline & Freq. \% & $\begin{array}{l}\text { Mean } \\
\text { Cover }\end{array}$ & I.V. & Freq.\% & $\begin{array}{l}\text { Mean } \\
\text { Cover }\end{array}$ & I.V. \\
\hline Sporobolus clandestinus & 100 & 28.20 & 46.1 & -- & -- & -- \\
\hline Selaginella rupestris & 68 & 12.37 & 22.4 & 66 & 15.36 & 25.1 \\
\hline Koeleria macrantha & 86 & 8.16 & 18.3 & 32 & 2.36 & 6.3 \\
\hline Ambrosia psilostachya & 98 & 6.97 & 17.9 & 84 & 7.31 & 17.8 \\
\hline Asclepias verticillata & 96 & 5.06 & 15.3 & 28 & 0.63 & 3.9 \\
\hline Cyperus lupulinus & 92 & 0.66 & 9.1 & 76 & 1.66 & 10.5 \\
\hline Opuntia macrorhiza & 52 & 3.08 & 8.8 & 80 & 16.53 & 28.0 \\
\hline Leptoloma cognatum & 56 & 1.76 & 7.3 & 22 & 1.18 & 3.9 \\
\hline Cyperus schweinitzii & 66 & 0.82 & 7.0 & 16 & 0.28 & 2.1 \\
\hline Sporobolus cryptandrus & 54 & 1.10 & 6.3 & 8 & 0.04 & 1.0 \\
\hline Plantago patagonica & 62 & 0.31 & 6.0 & 10 & 0.05 & 1.2 \\
\hline *Poa pratensis & 56 & 0.48 & 5.6 & 26 & 1.15 & 4.2 \\
\hline Physalis virginiana & 36 & 0.87 & 4.3 & 12 & 0.40 & 1.8 \\
\hline Dichanthelium villosissimum & 24 & 0.37 & 2.7 & 38 & 0.78 & 5.2 \\
\hline Monarda punctata & 12 & 1.03 & 2.5 & -- & -- & -- \\
\hline Dichanthelium oligosanthes & 20 & 0.30 & 2.2 & 2 & 0.01 & 0.2 \\
\hline Lithospermum croceum & 18 & 0.43 & 2.2 & 12 & 0.21 & 1.5 \\
\hline Oenothera clelandii & 22 & 0.16 & 2.2 & -- & -- & -- \\
\hline Rhus aromatica & 2 & 1.25 & 1.9 & -- & -- & -- \\
\hline *Achillea millefolium & 10 & 0.44 & 1.5 & -- & -- & -- \\
\hline Panicum virgatum & 12 & 0.16 & 1.3 & 20 & 0.30 & 2.6 \\
\hline Schizachyrium scoparium & 4 & 0.60 & 1.2 & 82 & 8.68 & 19.2 \\
\hline Paspalum setaceum & 10 & 0.10 & 1.0 & -- & -- & -- \\
\hline Solidago nemoralis & 6 & 0.37 & 1.0 & -- & -- & -- \\
\hline Carex muhlenbergii & 8 & 0.04 & 0.8 & 22 & 0.41 & 3.0 \\
\hline Lespedeza capitata & 8 & 0.04 & 0.8 & -- & -- & -- \\
\hline *Potentilla recta & 6 & 0.08 & 0.6 & -- & -- & -- \\
\hline Sorghastrum nutans & 6 & 0.08 & 0.6 & -- & -- & -- \\
\hline Verbena stricta & 4 & 0.12 & 0.6 & -- & -- & -- \\
\hline Eragrostis spectabilis & 4 & 0.07 & 0.5 & -- & -- & -- \\
\hline Penstemon pallidus & 4 & 0.02 & 0.4 & -- & -- & -- \\
\hline Physalis heterophylla & 2 & 0.06 & 0.3 & -- & -- & -- \\
\hline Sporobolus compositus & 2 & 0.06 & 0.3 & -- & -- & -- \\
\hline Antennaria neglecta & 2 & 0.01 & 0.2 & -- & -- & -- \\
\hline Aristida basiramea & 2 & 0.01 & 0.2 & -- & -- & -- \\
\hline Bouteloua hirsuta & 2 & 0.01 & 0.2 & 12 & 0.45 & 1.8 \\
\hline Oxalis stricta & 2 & 0.01 & 0.2 & -- & -- & -- \\
\hline Psuedognaphalium obtusifolium & 2 & 0.01 & 0.2 & -- & -- & -- \\
\hline Heterostipa spartea & -- & -- & -- & 98 & 16.72 & 30.3 \\
\hline Tephrosia virginiana & -- & -- & -- & 28 & 5.52 & 9.6 \\
\hline Carex tonsa & -- & -- & -- & 24 & 0.17 & 2.9 \\
\hline Equisetum laevigatum & -- & -- & -- & 24 & 0.12 & 2.8 \\
\hline Brickellia eupatorioides & -- & -- & -- & 12 & 0.98 & 2.4 \\
\hline Callirhoe triangulata & -- & -- & -- & 4 & 1.55 & 2.3 \\
\hline Andropogon gerardii & -- & -- & -- & 8 & 0.96 & 2.0 \\
\hline Ceanothus herbaceus & -- & -- & -- & 4 & 1.26 & 2.0 \\
\hline Croton glandulosus & -- & -- & -- & 10 & 0.79 & 2.0 \\
\hline Euphorbia corollata & -- & -- & -- & 10 & 0.39 & 1.5 \\
\hline Helianthus pauciflorus & -- & -- & -- & 6 & 0.18 & 0.9 \\
\hline Paspalum bushii & -- & -- & -- & 4 & 0.36 & 0.9 \\
\hline Chrysopsis camporum & -- & -- & -- & 6 & 0.03 & 0.7 \\
\hline *Chenopodium album & -- & -- & -- & 2 & 0.01 & 0.2 \\
\hline Solanum carolinense & -- & -- & -- & 2 & 0.01 & 0.2 \\
\hline Totals & & 75.67 & 200.0 & & 86.84 & 200.0 \\
\hline Bare ground and litter & & 22.36 & & & 22.99 & \\
\hline
\end{tabular}


Table 4. Frequency (\%), mean cover (\% of total area), and importance value (I.V.) of the ground layer species encountered in the fall of 2005 in burned and unburned mature dry sand prairie communities at Lost Mound, Jo Daviess County, Illinois. (*non-native species)

\begin{tabular}{|c|c|c|c|c|c|c|}
\hline \multirow[b]{2}{*}{ Species } & \multicolumn{3}{|c|}{$\begin{array}{c}\text { Unburned Dry Sand Prairie Community } \\
\text { Area } 8(n=50)\end{array}$} & \multicolumn{3}{|c|}{$\begin{array}{c}\text { Burned Dry Sand Prairie Community } \\
\text { Area } 9(\mathrm{n}=50)\end{array}$} \\
\hline & Freq. $\%$ & Mean Cover & I.V. & Freq. $\%$ & Mean Cover & I.V. \\
\hline Schizachyrium scoparium & 96 & 21.30 & 40.4 & 90 & 5.42 & 16.2 \\
\hline Selaginella rupestris & 78 & 10.94 & 23.2 & 24 & 0.56 & 2.9 \\
\hline Ambrosia psilostachya & 86 & 8.43 & 20.1 & 88 & 9.56 & 22.7 \\
\hline *Rumex acetosella & 78 & 1.58 & 9.1 & 98 & 8.85 & 22.5 \\
\hline *Potentilla recta & 70 & 1.92 & 9.0 & 30 & 1.47 & 4.9 \\
\hline Andropogon gerardii & 22 & 4.59 & 8.8 & 14 & 2.07 & 4.5 \\
\hline Cyperus lupulinus & 80 & 1.15 & 8.6 & 60 & 0.55 & 5.8 \\
\hline Koeleria macrantha & 64 & 1.60 & 7.9 & 66 & 2.83 & 10.0 \\
\hline Carex tonsa & 62 & 0.86 & 6.7 & 42 & 0.31 & 4.0 \\
\hline Leptoloma cognatum & 50 & 1.52 & 6.6 & 54 & 3.34 & 9.8 \\
\hline *Poa pratensis & 46 & 1.69 & 6.6 & 24 & 0.41 & 2.7 \\
\hline Opuntia macrorhiza & 26 & 2.81 & 6.4 & 34 & 3.19 & 8.0 \\
\hline Sorghastrum nutans & 22 & 1.63 & 4.4 & 14 & 0.27 & 1.5 \\
\hline Dichanthelium villosissimum & 36 & 0.77 & 4.3 & 46 & 0.38 & 4.4 \\
\hline *Achillea millefolium & 36 & 0.38 & 3.7 & 46 & 0.68 & 4.9 \\
\hline Carex muhlenbergii & 36 & 0.28 & 3.5 & 14 & 0.07 & 1.2 \\
\hline Asclepias verticillata & 36 & 0.18 & 3.4 & 68 & 0.49 & 6.4 \\
\hline Bouteloua hirsuta & 18 & 1.11 & 3.3 & 4 & 0.07 & 0.4 \\
\hline Physalis virginiana & 28 & 0.54 & 3.2 & 24 & 0.27 & 2.4 \\
\hline Solidago nemoralis & 16 & 0.81 & 2.6 & 18 & 0.97 & 3.0 \\
\hline Lithospermum croceum & 20 & 0.40 & 2.3 & 14 & 1.38 & 3.3 \\
\hline Polygala polygama & 22 & 0.11 & 2.1 & 28 & 0.24 & 2.7 \\
\hline Dichanthelium oligosanthes & 20 & 0.20 & 2.0 & 8 & 0.04 & 0.8 \\
\hline Plantago patagonica & 20 & 0.10 & 1.9 & -- & -- & -- \\
\hline Monarda punctata & 12 & 0.45 & 1.7 & 2 & 0.06 & 0.3 \\
\hline Oenothera clelandii & 14 & 0.12 & 1.4 & 16 & 0.18 & 1.6 \\
\hline Aster ericoides & 8 & 0.19 & 1.0 & 60 & 4.33 & 11.9 \\
\hline Sporobolus cryptandrus & 10 & 0.05 & 1.0 & -- & -- & -- \\
\hline Rhus aromatica & 2 & 0.30 & 0.7 & 12 & 1.93 & 4.1 \\
\hline Conyza canadensis & 6 & 0.03 & 0.5 & -- & -- & -- \\
\hline Aristida basiramea & 4 & 0.02 & 0.3 & -- & -- & -- \\
\hline Aristida tuberculosa & 4 & 0.02 & 0.3 & -- & -- & -- \\
\hline Cyperus schweinitzii & 4 & 0.02 & 0.3 & 18 & 0.09 & 1.5 \\
\hline Helianthemum canadense & 2 & 0.06 & 0.3 & -- & -- & -- \\
\hline Panicum virgatum & 4 & 0.02 & 0.3 & -- & -- & -- \\
\hline *Potentilla argentea & 2 & 0.06 & 0.3 & -- & -- & -- \\
\hline Croton glandulosus & 2 & 0.01 & 0.2 & 2 & 0.01 & 0.2 \\
\hline Dichanthelium perlongum & 2 & 0.01 & 0.2 & 2 & 0.01 & 0.2 \\
\hline Draba reptans & 2 & 0.01 & 0.2 & -- & -- & -- \\
\hline Gleditsia triacanthos & 2 & 0.01 & 0.2 & -- & -- & -- \\
\hline Hieracium longipilum & 2 & 0.01 & 0.2 & -- & -- & -- \\
\hline Liatris aspera & 2 & 0.01 & 0.2 & -- & -- & -- \\
\hline Linum sulcatum & 2 & 0.01 & 0.2 & 2 & 0.01 & 0.2 \\
\hline Oxalis stricta & 2 & 0.01 & 0.2 & 30 & 0.35 & 3.1 \\
\hline *Poa compressa & 2 & 0.01 & 0.2 & -- & -- & -- \\
\hline Amorpha canescens & -- & -- & -- & 46 & 2.96 & 8.6 \\
\hline Tephrosia virginiana & -- & -- & -- & 16 & 2.16 & 4.8 \\
\hline Ionactis linariifolius & -- & -- & -- & 18 & 1.90 & 4.5 \\
\hline Helianthus occidentalis & -- & -- & -- & 18 & 1.16 & 3.3 \\
\hline Coreopsis palmata & -- & -- & -- & 8 & 0.72 & 1.9 \\
\hline Aster sericeus & -- & -- & -- & 8 & 0.67 & 1.8 \\
\hline Anemone cylindrica & -- & -- & -- & 8 & 0.38 & 1.3 \\
\hline Callirhoe triangulata & -- & -- & -- & 6 & 0.42 & 1.2 \\
\hline Eragrostis spectabilis & -- & -- & -- & 10 & 0.20 & 1.1 \\
\hline Ceanothus americanus & -- & -- & -- & 2 & 0.30 & 0.7 \\
\hline Heterostipa spartea & -- & -- & -- & 6 & 0.13 & 0.7 \\
\hline Physalis subglabrata & -- & -- & -- & 4 & 0.12 & 0.5 \\
\hline Bouteloua curtipendula & -- & -- & -- & 2 & 0.06 & 0.3 \\
\hline Equisetum laevigatum & -- & -- & -- & 2 & 0.06 & 0.3 \\
\hline Froelichia gracilis & -- & -- & -- & 4 & 0.02 & 0.3 \\
\hline Asclepias viridiflora & -- & -- & -- & 2 & 0.01 & 0.2 \\
\hline Dalea purpurea & -- & -- & -- & 2 & 0.01 & 0.2 \\
\hline Solanum carolinense & -- & -- & -- & 2 & 0.01 & 0.2 \\
\hline Totals & & 66.33 & 200.0 & & 61.68 & 200.0 \\
\hline Bare ground and litter & & 26.70 & & & 38.10 & \\
\hline
\end{tabular}


Table 5. Size class density (\#/ha), basal area $\left(\mathrm{m}^{2} / \mathrm{ha}\right)$, relative values, importance value (I.V.), and average diameter $(\mathrm{cm})$ of the woody species encountered in 2005 in a dry sand savanna associated with a blowout at Lost Mound, Jo Daviess County, Illinois. (*non-native species)

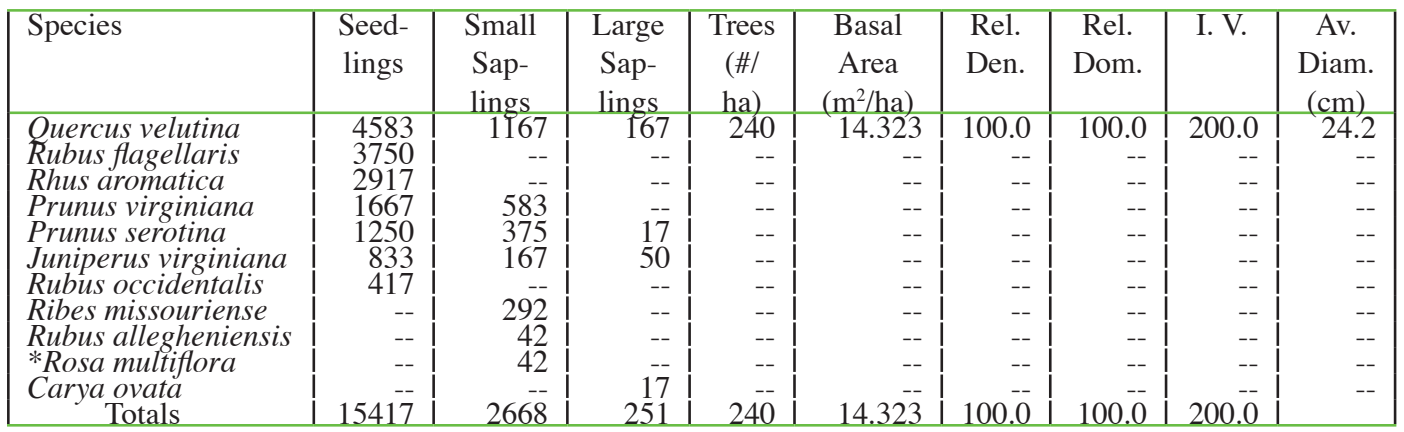

\section{Ayers Sand Prairie Nature Preserve}

A total of 175 species in 132 genera and 56 families was documented (Appendix I). Ferns, ferns-allies, and gymnosperms accounted for 4 species, while 42 were monocots in 4 families and 28 genera, and 129 were dicots in 48 families and 100 genera. Adventive species accounted for 36 taxa, about $20 \%$ of all species. The state-threatened (Herkert and Ebinger 2002) Cyperus grayoides was a common associate of blowouts. The FQI for this site when adventive species were included was 47.62 with a mean $\mathrm{C}$-value of 3.60, and with the adventive species excluded from the calculations the FQI was 52.73 with a mean C-value of 4.41 .

\section{Blowing Sand Community (early succes-}

sional): In areas of blowing sand, plants were widely scattered and bare ground and litter averaged $61 \%$ cover. Numerous species were established in these areas with Aristida tuberculosa (IV of 32.1), Dichanthelium villosissimum (IV of 27.3) and Ambrosia psilostachya (IV of 17.9) the most common. Most of the species associated with the mature and disturbed dry sand prairie were also found, but in low numbers (Table 9). A few species, such as Carex tonsa (shaved sedge), Callirhoe triangulata (poppy mallow), Viola pedata (bird's-foot violet), $C y$ perus schweinitzii, Liatris aspera (rough blazing-star), Polygonella articulata (jointweed), and Chamaesyce geyeri (Geyer's spurge) were more common in these areas of blowing sand than in the mature or disturbed sand prairies (Table 9).

Dry Sand Prairie Community (mid-successional): The disturbed dry sand prairie community had a high species diversity that included many taxa associated with dry sand prairies. Two bunch-grasses, Dichanthelium villosissimum and Koeleria macrantha, dominated this community with IVs of 37.0 and 30.1, respectively (Table 9). The disturbance species Croton glandulosus (IV of 15.5) and Aristida tuberculosa (IV of 13.2) were third and forth in IV, followed by Ambrosia psilostachya and Hudsonia tomentosa. Bare ground and litter averaged $46 \%$ cover.

\section{Dry Sand Prairie Community (mature or} late successional): In the mature dry sand prairie Schizachyrium scoparium dominated with an IV of 52.5 and a mean cover of 31.4 (Table 9). Ambrosia psilostachya was second with an IV of 26.4, followed by Solidago nemoralis (IV of 14.1), and Koeleria macrantha (IV of 13.0). The remaining 40 species encountered in the plots mostly had low frequencies and mean covers. The grasses formed extensive clumps while most other species grew in spaces between clumps, and were referred to as interstitial species by Gleason (1910). The exotic species Achillea millefolium, Mollugo verticillata, and Poa pratensis were rare. Bare ground and litter averaged $28 \%$ cover (Table 9 ). 
Table 6. Size class density (\#/ha), basal area $\left(\mathrm{m}^{2} / \mathrm{ha}\right)$, relative values, importance value (I.V.), and average diameter $(\mathrm{cm})$ of the woody species encountered in 2005 in a mature second growth dry upland sand forest community associated with dune topography at Lost Mound, Jo Daviess County, Illinois. (*non-native species)

\begin{tabular}{|c|c|c|c|c|c|c|c|c|c|}
\hline Species & $\begin{array}{l}\text { Seed- } \\
\text { lings }\end{array}$ & $\begin{array}{c}\text { Small } \\
\text { Sap- } \\
\text { lings }\end{array}$ & $\begin{array}{l}\text { Large } \\
\text { Sap- } \\
\text { lings }\end{array}$ & $\begin{array}{c}\text { Trees } \\
\text { (\#/ } \\
\text { ha) }\end{array}$ & $\begin{array}{l}\text { Basal } \\
\text { Area } \\
\left(\mathrm{m}^{2} / \mathrm{ha}\right)\end{array}$ & $\begin{array}{l}\text { Rel. } \\
\text { Den. }\end{array}$ & $\begin{array}{l}\text { Rel. } \\
\text { Dom. }\end{array}$ & I. V. & $\begin{array}{c}\text { Av. } \\
\text { Diam. } \\
(\mathrm{cm})\end{array}$ \\
\hline $\begin{array}{l}\text { Quercus velutina } \\
\text { Prunus serotina }\end{array}$ & $\begin{array}{l}3125 \\
1094\end{array}$ & $\begin{array}{l}125 \\
750\end{array}$ & 35 & 332 & $\begin{array}{r}22.599 \\
0.310\end{array}$ & $\begin{array}{r}92.2 \\
6.6\end{array}$ & 98.4 & $\begin{array}{l}190.6 \\
8.0\end{array}$ & $\begin{array}{l}26.9 \\
127\end{array}$ \\
\hline & 313 & 188 & 13 & & 0.025 & 0.6 & 0.1 & $\begin{array}{l}0.5 \\
0.4\end{array}$ & 12.6 \\
\hline Juglar & 17180 & 2812 & & & 0.010 & 0.3 & & 0.3 & 11.4 \\
\hline $\begin{array}{l}\text { Rubus all } \\
\text { Cornus ra }\end{array}$ & $\begin{array}{r}1188 \\
4688\end{array}$ & $\begin{array}{l}2813 \\
1844\end{array}$ & 6 & -- & -- & -- & $\begin{array}{l}-- \\
--\end{array}$ & -- & $\begin{array}{l}-- \\
--\end{array}$ \\
\hline Prunus vi & $\begin{array}{l}1875 \\
1406\end{array}$ & 2688 & 6 & -- & -- & -- & -- & -- & -- \\
\hline Rubus & 250 & 812 & -- & -- & -- & -- & -- & -- & -- \\
\hline $\begin{array}{l}\text { Ribes m } \\
\text { Gledits }\end{array}$ & $\begin{array}{l}1094 \\
938\end{array}$ & $\begin{array}{l}406 \\
125\end{array}$ & 6 & -- & -- & -- & -- & -- & -- \\
\hline & 313 & 194 & 31 & 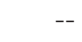 & 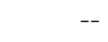 & , & & 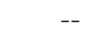 & -- \\
\hline & 313 & & & & & - & & & -- \\
\hline & & & - & & -- & -- & -- & -- & -- \\
\hline Junl & - & 94 & & & -- & -- & -- & -- & -- \\
\hline $\begin{array}{l}\text { Ulmus americana } \\
\text { Totals }\end{array}$ & & 10533 & $\begin{array}{r}6 \\
468\end{array}$ & 360 & 22.959 & $100 . \overline{0}$ & 100.0 & 200.0 & \\
\hline
\end{tabular}

Table 7. Size class density (\#/ha), basal area $\left(\mathrm{m}^{2} / \mathrm{ha}\right)$, relative values, importance value (I.V.), and average diameter $(\mathrm{cm})$ of the woody species encountered in 2005 in a mature second growth drymesic upland sand forest at Lost Mound, Jo Daviess County, Illinois. (*non-native species)

\begin{tabular}{|c|c|c|c|c|c|c|c|c|c|}
\hline Species & $\begin{array}{l}\text { Seed- } \\
\text { lings }\end{array}$ & $\begin{array}{l}\text { Small } \\
\text { Sap- } \\
\text { lings }\end{array}$ & $\begin{array}{l}\text { Large } \\
\text { Sap- } \\
\text { lings }\end{array}$ & $\begin{array}{c}\text { Trees } \\
(\# / \\
\text { ha) }\end{array}$ & $\begin{array}{l}\text { Basal } \\
\text { Area } \\
\left(\mathrm{m}^{2} /\right.\end{array}$ & $\begin{array}{l}\text { Rel. } \\
\text { Den. }\end{array}$ & $\begin{array}{l}\text { Rel. } \\
\text { Dom. }\end{array}$ & I. V. & $\begin{array}{c}\text { Av. } \\
\text { Diam. } \\
(\mathrm{cm})\end{array}$ \\
\hline $\begin{array}{l}\text { Quercus alba } \\
\text { Quercus velutina } \\
\text { Carya cordiformis } \\
\text { Prunus serotina } \\
\text { Ulmus americana } \\
\text { Ulmus rubra } \\
\text { Celtis occidentalis } \\
\text { *Robinia pseudoacacia } \\
\text { Tilia americana } \\
\text { Ouercus rubra } \\
\text { Betula nigra } \\
\text { Carya ovata } \\
\text { Juglans cinerea } \\
\text { *Morus alba } \\
\text { Rubus allegheniensis } \\
\text { Ribes missouriense } \\
\text { Gleditsia triacanthos } \\
\text { Zanthoxylum americanum } \\
\text { Cornus racemosa } \\
\text { Rubus occidentalis } \\
\text { Juniperus virginiana } \\
\text { Celastrus scandens } \\
\text { *Lonicera tatarica } \\
\text { Corylus americana } \\
\text { *Rosa multiflora } \\
\text { Acer negundo } \\
\text { Totals }\end{array}$ & $\begin{array}{r}-- \\
4063 \\
1719 \\
781 \\
625 \\
469 \\
469 \\
313 \\
156 \\
156 \\
-- \\
-- \\
30158\end{array}$ & $\begin{array}{r}-- \\
359 \\
47 \\
47 \\
413 \\
94 \\
-- \\
-- \\
16 \\
47\end{array}$ & $\begin{array}{r}188 \\
331 \\
38 \\
94 \\
63 \\
63 \\
38\end{array}$ & $\begin{array}{r}99 \\
78 \\
63 \\
38 \\
20 \\
15 \\
11 \\
10 \\
7 \\
3 \\
1 \\
1 \\
- \\
-- \\
-- \\
--\end{array}$ & $\begin{array}{r}14.086 \\
9.352 \\
.964 \\
.689 \\
.448 \\
.308 \\
.275 \\
.107 \\
.150 \\
.017 \\
.054 \\
.015 \\
.025 \\
--\end{array}$ & $\begin{array}{r}28.3 \\
22.3 \\
18.0 \\
10.9 \\
5.7 \\
4.2 \\
3.1 \\
2.8 \\
2.0 \\
0.9 \\
0.9 \\
0.3 \\
0.3 \\
0.3\end{array}$ & $\begin{array}{r}52.6 \\
34.9 \\
3.6 \\
2.6 \\
1.7 \\
1.2 \\
1.0 \\
0.4 \\
0.6 \\
0.8 \\
0.2 \\
0.2 \\
0.1 \\
0.1\end{array}$ & $\begin{array}{r}80.9 \\
57.2 \\
21.6 \\
13.5 \\
7.4 \\
5.4 \\
4.1 \\
3.2 \\
2.6 \\
1.7 \\
1.1 \\
0.5 \\
0.4 \\
0.4\end{array}$ & $\begin{array}{r}40.0 \\
37.7 \\
13.4 \\
14.5 \\
16.0 \\
15.7 \\
17.0 \\
11.7 \\
16.3 \\
14.8 \\
29.6 \\
13.6 \\
18.0 \\
-- \\
-- \\
-- \\
-- \\
-- \\
-- \\
-- \\
--\end{array}$ \\
\hline
\end{tabular}




\section{Thompson-Fulton Sand Prairie Nature Preserve}

A total of 182 species in 133 genera and 54 families was documented (Appendix I). Ferns, fern-allies, and gymnosperms accounted for 5 species, while 42 were monocots in 4 families and 28 genera, and 135 were dicots in 46 families and 101 genera. Adventive species accounted for 38 taxa, about $20 \%$ of all species. The state-endangered (Herkert and Ebinger 2002) Penstemon grandiflorus (large-flowered beardstongue) was relatively common in a small part of the preserve, while the statethreatened Cyperus grayoides was occasionally encountered. The FQI for this site when adventive species were included was 46.81 with a mean C-value of 3.47, and with the adventive species excluded from the calculations the FQI was 52.86 with a mean C-value of 4.42 .

\section{Dry Sand Prairie Community (early succes-} sional): The disturbance community contained many species commonly encountered in dry sand prairies. The most important forbs of this community were Opuntia macrorhiza (IV of 31.6) and Ambrosia psilostachya (IV of 24.8). The important grasses included the two bunch-grasses Koeleria macrantha (IV of 21.2) and Dichanthelium villosissimum (IV of 12.7) along with the common disturbance area grass Aristida tuberculosa (IV of 23.6)(Table 10). Schizachyrium scoparium was scarce; only a few scattered individuals were observed and none of these were found in plots. Three adventive species (Rumex acetosella, Mollugo verticillata, Bromus tectorum) were encountered in the plots, all with IV's of 1.7 or lower. Bare ground and litter mean cover was $7 \%$ (Table 10).

\section{Dry Sand Prairie Community (mature or} late successional): Schizachyrium scoparium, the leading dominant of the mature sand prairie, had an IV of 39.4 and a mean cover of $20.9 \%$ (Table 10). Opuntia macrorhiza was second with an IV of 31.7, followed by Ambrosia psilostachya (IV of 26.4), Tephrosia virginiana (IV of 21.9), and Dichanthelium villosissimum (IV of 21.8). Except for Tephrosia virginiana, which generally had a clumped distribution, these five species had frequencies of 84-91\% with a mean cover higher than $8.0 \%$ (Table 10). Schizachyrium scoparium and D. villosissimum grew in clumps $10-40 \mathrm{~cm}$ across, forming the bunch-grass association described by Gleason (1910). Most other species grew in spaces between clumps. Of the remaining 24 species encountered in the plots, most had frequencies of less than $50 \%$ and IVs lower than 8.0. The exotic species Rumex acetosella was rare, while bare ground and litter mean cover was $22 \%$ (Table 10).

\section{Big River State Forest}

A total of 162 species in 127 genera and 54 families was documented (Appendix I). Gymnosperms accounted for 2 species, while 41 were monocots in 5 families and 27 genera, and 162 were dicots in 54 families and 127 genera. Adventive species accounted for 37 taxa, about $20 \%$ of all species. The state-endangered (Herkert and Ebinger 2002) Penstemon grandiflorus and Stylisma pickeringii (Patterson bindweed) were encountered in the dry sand prairie. The FQI for this site when adventive species were included was 38.18 with a mean $\mathrm{C}$-value of 3.00, and with the adventive species excluded from the calculations the FQI was 43.47 with a mean C-value of 3.89.

\section{Dry Sand Prairie Community (mature or} late successional): The leading dominant of the mature sand prairie was Schizachyrium scoparium with an IV of 41.9 and a mean cover of $34 \%$ (Table 11). This species formed extensive clumps, many more than $40 \mathrm{~cm}$ across, while most other taxa were interstitial species. Solidago nemoralis (gray goldenrod) was second with an IV of 24.3, followed by Opuntia macrorhiza (IV of 19.0), and Ambrosia psilostachya (IV of 18.4). Lespedeza capitata (round-headed bush clover), Stylisma pickeringii, and Monarda punctata (horsemint) had IVs exceeding 10, while Dichanthelium villosissimum, Cyperus lupulinus, and Commelina erecta (day flower) had frequencies greater than $75 \%$ (Table 11). Most of the remaining species encountered had frequencies of less than 50\% and IV's lower than 5.0. The only exotic species in the plots, Poa pratensis and Chenopodium album, were rare, having an IV of 0.2. Bare ground and litter had a mean cover of $10.7 \%$, though in some areas the herbaceous vine, Stylisma pickeringii completely covered the plots (Table 11). 
Table 8. Size class density (\#/ha), basal area $\left(\mathrm{m}^{2} / \mathrm{ha}\right)$, relative values, importance value (I.V.), and average diameter $(\mathrm{cm})$ of the woody species encountered in 2005 in a wet-mesic floodplain forest at the edge of the Mississippi River, Lost Mound, Jo Daviess County, Illinois. (*non-native species)

\begin{tabular}{|c|c|c|c|c|c|c|c|c|c|}
\hline Species & $\begin{array}{l}\text { Seed- } \\
\text { lings }\end{array}$ & $\begin{array}{l}\text { Small } \\
\text { Sap- } \\
\text { lings }\end{array}$ & $\begin{array}{c}\text { Large } \\
\text { Sap- } \\
\text { lings }\end{array}$ & $\begin{array}{l}\text { Trees } \\
(\# / \text { ha) }\end{array}$ & $\begin{array}{l}\text { Basal } \\
\text { Area } \\
\left(\mathrm{m}^{2} /\right.\end{array}$ & $\begin{array}{l}\text { Rel. } \\
\text { Den. }\end{array}$ & $\begin{array}{l}\text { Rel. } \\
\text { Dom. }\end{array}$ & I. V. & $\begin{array}{c}\text { Av. } \\
\text { Diam. } \\
(\mathrm{cm})\end{array}$ \\
\hline $\begin{array}{l}\text { Acer saccharinum } \\
\text { Ulmus americana } \\
\text { Fraxinus lanceolata } \\
\text { Celtis occidentalis } \\
* \text { Morus alba } \\
\text { Totals }\end{array}$ & $\begin{array}{r}80938 \\
21563 \\
7813 \\
156 \\
110470\end{array}$ & $\begin{array}{r}344 \\
156 \\
-- \\
-- \\
500\end{array}$ & $\begin{array}{l}6 \\
-- \\
-- \\
--\end{array}$ & $\begin{array}{r}217 \\
25 \\
4 \\
1\end{array}$ & $\begin{array}{r}34.175 \\
1.151 \\
0.596 \\
0.026 \\
35.948\end{array}$ & $\begin{array}{r}87.9 \\
10.1 \\
1.6 \\
0.4\end{array}$ & $\begin{array}{r}95.0 \\
3.2 \\
1.7 \\
0.1 \\
100.0\end{array}$ & $\begin{array}{r}182.9 \\
13.3 \\
3.3 \\
0.5 \\
200.0\end{array}$ & $\begin{array}{l}41.7 \\
23.5 \\
23.5 \\
18.2\end{array}$ \\
\hline
\end{tabular}

Dry Sand Savanna Community (degraded): Dry sand forest occurs just to the north of the dry sand prairie and continues for more than 1 $\mathrm{km}$. Probably clear-cut soon after settlement, this forest has also been subjected to more recent cutting and fire suppression. Quercus velutina and $Q$. marilandica (blackjack oak) dominated this degraded sand savanna, which, due to fire suppression, is now a closed canopy forest. On the site trees averaged 588 stems/ha with an average basal area of $17.324 \mathrm{~m}^{2} / \mathrm{ha}$ (Table 12). The oaks averaged 17.3 to $18.7 \mathrm{~cm}$ $\mathrm{dbh}$, and except for a few Juniperus virginiana (red cedar) and Prunus serotina, were the only species that reached tree size $(\geq 10 \mathrm{~cm} \mathrm{dbh})$. The seedling and sapling layers were dense; woody seedlings averaged 19,376 stems/ha, small saplings averaged 11,187 stems/ha, but large saplings averaged only 318 stems/ha (Table 12). Black oak dominated the seedling layer (7,500 stems/ha) and was second in small saplings (1,594 stems/ha) and large saplings (106 stems/ha). Blackjack oak was first in large saplings with 131 stems/ha. Species of Rubus (blackberries and raspberries) and Cornus drummondii (rough-leaved dogwood) were very common components of the seedling and small sapling layers.

\section{DATA ANALYSIS AND SITE SIMILARITY}

A summary of the floristic data and the Floristic Quality Index for each of the 15 prairie study sites ( 9 transects at Lost Mound, 3 at Ayers Nature Preserve, 2 at Thomson-Fulton Nature Preserve, and 1 at Big River State Forest) are included in Table 13. In this table the 15 study sites are grouped by the amount of past and present disturbances and the extent to which Schizachyrium scoparium dominated each community. Throughout the dry sand prairies of the Mississippi River valley in northwestern Illinois, Schizachyrium scoparium is usually one of the dominant species, although its importance decreased in successional and disturbance communities. Among all sites, native species richness ranged from 22 to 46 while adventive species richness was low, ranging from 1 to 7 species; the percent of native taxa exceeded 90\% at all but two sites (Table 13). Little variation occurs in the Floristic Quality Index (FQI) of the sites (Table 13). The FQI for the sites ranged from 20.74 to 35.07 , with only two exceeding 30.

Within the Mississippi River sand deposits, many of the sand prairie communities studied had a relatively high degree of similarity (Table 14). The Sorensen Indices of Similarity (ISs) for the 15 sand prairie areas examined ranged from $35.6 \%$ to $83.9 \%$ with most values above $50 \%$. The lowest ISs was between the blowout community (Area 1) and the burned dry sand prairie (Area 9), both at Lost Mound. The highest ISs was between the blowing sand community at Ayers Nature Preserve (Area 12) and the successional dry sand prairie at Thomson/Fulton Nature Preserve (Area 14). All communities at Ayers Nature Preserve and Thomson-Fulton Nature Preserve were very similar as shown by the constantly high ISs, which ranged from 59.3 to 83.9 (Table 14). Overall, the vegetation of the mature dry sand prairie at Big River State Forest had a slightly lower similarity to Continued on page 209 
Table 9. Frequency (\%), mean cover (\% of total cover), and importance value (I.V.) of the ground layer species encountered in 2004 in three plant communities at Ayers Nature Preserve, Carroll County, Illinois. (*non-native species)

\begin{tabular}{|c|c|c|c|c|c|c|c|c|c|}
\hline \multirow[b]{2}{*}{ Species } & \multicolumn{3}{|c|}{$\begin{array}{c}\text { Dry Sand Prairie } \\
\text { (late successional) } \\
\text { Area } 10(\mathrm{n}=50)\end{array}$} & \multicolumn{3}{|c|}{$\begin{array}{c}\text { Dry Sand prairie } \\
\text { (mid-successional) } \\
\text { Area } 11(\mathrm{n}=50)\end{array}$} & \multicolumn{3}{|c|}{$\begin{array}{c}\text { Blowing Sand } \\
\text { (early successional) } \\
\text { Area } 12(\mathrm{n}=50)\end{array}$} \\
\hline & Freq. $\%$ & $\begin{array}{l}\text { Mean } \\
\text { Cover }\end{array}$ & I. $\mathrm{V}$ & Freq. $\%$ & $\begin{array}{l}\text { Mean } \\
\text { Cover }\end{array}$ & I. V. & Freq. $\%$ & $\begin{array}{l}\text { Mean } \\
\text { Cover. }\end{array}$ & I. V. \\
\hline Schizachyrium scoparium & 100 & 31.40 & 52.5 & 2 & 0.01 & 0.2 & -- & -- & -- \\
\hline Ambrosia psilostachya & 98 & 12.54 & 26.4 & 86 & 2.45 & 12.5 & 52 & 4.48 & 17.9 \\
\hline Solidago nemoralis & 76 & 5.18 & 14.1 & 8 & 0.14 & 1.0 & 2 & 0.30 & 1.0 \\
\hline Koeleria macrantha & 84 & 3.79 & 13.0 & 100 & 10.68 & 30.1 & 56 & 2.42 & 12.8 \\
\hline Dichanthelium villosissimum & 72 & 2.08 & 9.6 & 100 & 14.16 & 37.0 & 84 & 6.60 & 27.3 \\
\hline Carex muhlenbergii & 82 & 1.16 & 9.2 & 12 & 0.21 & 1.5 & 52 & 0.51 & 7.4 \\
\hline Asclepias verticillata & 72 & 1.35 & 8.6 & -- & -- & -- & 2 & 0.06 & 0.4 \\
\hline Carex tonsa & 56 & 2.29 & 8.3 & 60 & 1.73 & 8.8 & 48 & 3.30 & 14.3 \\
\hline Cyperus lupulinus & 68 & 0.94 & 7.6 & 4 & 0.02 & 0.4 & 8 & 0.04 & 1.0 \\
\hline Polygala polygama & 64 & 0.62 & 6.8 & 34 & 0.52 & 4.0 & 2 & 0.01 & 0.2 \\
\hline Aster ericoides & 26 & 2.60 & 6.0 & -- & -- & -- & -- & -- & -- \\
\hline Callirhoe triangulata & 22 & 2.02 & 4.8 & 4 & 0.36 & 1.1 & 18 & 2.67 & 9.2 \\
\hline Viola pedata & 22 & 0.99 & 3.4 & -- & -- & -- & 36 & 0.92 & 6.5 \\
\hline Conyza canadensis & 32 & 0.21 & 3.3 & 2 & 0.01 & 0.2 & 8 & 0.09 & 1.1 \\
\hline Lespedeza capitata & 20 & 0.88 & 3.0 & 4 & 0.07 & 0.5 & 4 & 0.36 & 1.4 \\
\hline Chrysopsis camporum & 16 & 0.81 & 2.6 & 6 & 0.13 & 0.8 & -- & -- & -- \\
\hline Panicum virgatum & 20 & 0.50 & 2.5 & -- & -- & -- & 4 & 0.02 & 0.6 \\
\hline Oenothera clelandii & 18 & 0.14 & 1.9 & 56 & 1.12 & 7.2 & 28 & 0.14 & 3.6 \\
\hline Lithospermum croceum & 10 & 0.49 & 1.6 & 2 & 0.01 & 0.2 & 2 & 0.01 & 0.2 \\
\hline Cyperus schweinitzii & 10 & 0.20 & 1.2 & 32 & 0.56 & 3.9 & 60 & 0.65 & 8.6 \\
\hline Hieracium longipilum & 10 & 0.20 & 1.2 & -- & -- & -- & -- & -- & -- \\
\hline Pseudognaphalium obtusifolium & 8 & 0.33 & 1.2 & -- & -- & -- & 2 & 0.06 & 0.4 \\
\hline *Achillea millefolium & 10 & 0.15 & 1.1 & -- & -- & -- & & -- & -- \\
\hline Chenopodium dessicatum & 10 & 0.05 & 1.0 & -- & -- & -- & 4 & 0.02 & 0.6 \\
\hline Draba reptans & 10 & 0.10 & 1.0 & 68 & 1.09 & 8.2 & -- & -- & -- \\
\hline Selaginella rupestris & 6 & 0.32 & 1.0 & -- & -- & -- & -- & -- & -- \\
\hline Leptoloma cognatum & 8 & 0.14 & 0.9 & 8 & 0.38 & 1.5 & 4 & 0.07 & 0.7 \\
\hline Liatris aspera & 8 & 0.14 & 0.9 & -- & -- & -- & 44 & 3.33 & 13.9 \\
\hline Plantago patagonica & 8 & 0.04 & 0.8 & 4 & 0.02 & 0.4 & -- & -- & -- \\
\hline Chamaecrista fasciculata & 6 & 0.03 & 0.6 & -- & -- & -- & -- & -- & -- \\
\hline Eragrostis spectabilis & 2 & 0.30 & 0.6 & -- & -- & -- & 2 & 0.06 & 0.4 \\
\hline Euphorbia corollata & 2 & 0.30 & 0.6 & 26 & 1.83 & 6.0 & 20 & 0.59 & 3.9 \\
\hline Physalis virginiana & 4 & 0.07 & 0.5 & 14 & 0.07 & 1.3 & 18 & 0.14 & 2.5 \\
\hline Aristida tuberculosa & 4 & 0.02 & 0.4 & 100 & 2.20 & 13.2 & 100 & 7.77 & 32.1 \\
\hline Chamaesyce geyeri & 4 & 0.02 & 0.4 & 12 & 0.16 & 1.4 & 30 & 0.25 & 4.1 \\
\hline Dichanthelium oligosanthes & 4 & 0.02 & 0.4 & -- & -- & -- & -- & -- & $--’$ \\
\hline *Mollugo verticillata & 4 & 0.02 & 0.4 & 60 & 0.70 & 6.7 & 22 & 0.11 & 2.8 \\
\hline Froelichia gracilis & 2 & 0.01 & 0.2 & -- & -- & -- & -- & -- & -- \\
\hline *Poa pratensis & 2 & 0.01 & 0.2 & -- & -- & -- & -- & -- & -- \\
\hline Polygonella articulata & 2 & 0.01 & 0.2 & 50 & 0.40 & 5.2 & 42 & 0.61 & 6.4 \\
\hline Croton glandulosus & -- & -- & -- & 100 & 3.38 & 15.5 & 48 & 0.44 & 6.7 \\
\hline Cyperus grayoides & -- & -- & -- & 86 & 1.58 & 10.8 & 46 & 0.58 & 6.8 \\
\hline Hudsonia tomentosa & -- & -- & -- & 42 & 3.48 & 10.6 & 2 & 0.30 & 1.0 \\
\hline Paspalum bushii & -- & -- & -- & 20 & 2.23 & 6.3 & 12 & 0.50 & 2.7 \\
\hline Diodia teres & -- & -- & -- & 8 & 0.19 & 1.1 & -- & -- & -- \\
\hline Monarda punctata & -- & -- & -- & 8 & 0.19 & 1.1 & -- & -- & -- \\
\hline Froelichia floridana & -- & -- & -- & 6 & 0.03 & 0.6 & 6 & 0.08 & 0.9 \\
\hline Asclepias viridiflora & -- & -- & -- & 2 & 0.06 & 0.3 & -- & -- & -- \\
\hline Cycloloma atriplicifolium & -- & -- & -- & 2 & 0.01 & 0.2 & -- & -- & -- \\
\hline Tradescantia ohiensis & -- & -- & -- & 2 & 0.01 & 0.2 & -- & -- & -- \\
\hline Rhus aromatica & -- & -- & -- & -- & -- & -- & 2 & 0.06 & 0.4 \\
\hline Apocynum sibericum & -- & -- & -- & -- & -- & -- & 2 & 0.01 & 0.2 \\
\hline Totals & & 72.47 & 200.0 & & 50.19 & 200.0 & & 37.56 & 200.0 \\
\hline Bare ground and litter & & 28.06 & & & 46.25 & & & 61.25 & \\
\hline
\end{tabular}


Table 10. Frequency (\%), mean cover (\% of total cover), and importance value (I.V.) of the ground layer species encountered in 2004 in mature dry sand prairie and disturbed dry sand prairie communities at Thomson-Fulton Nature Preserve, Whiteside County, Illinois. (*non-native species)

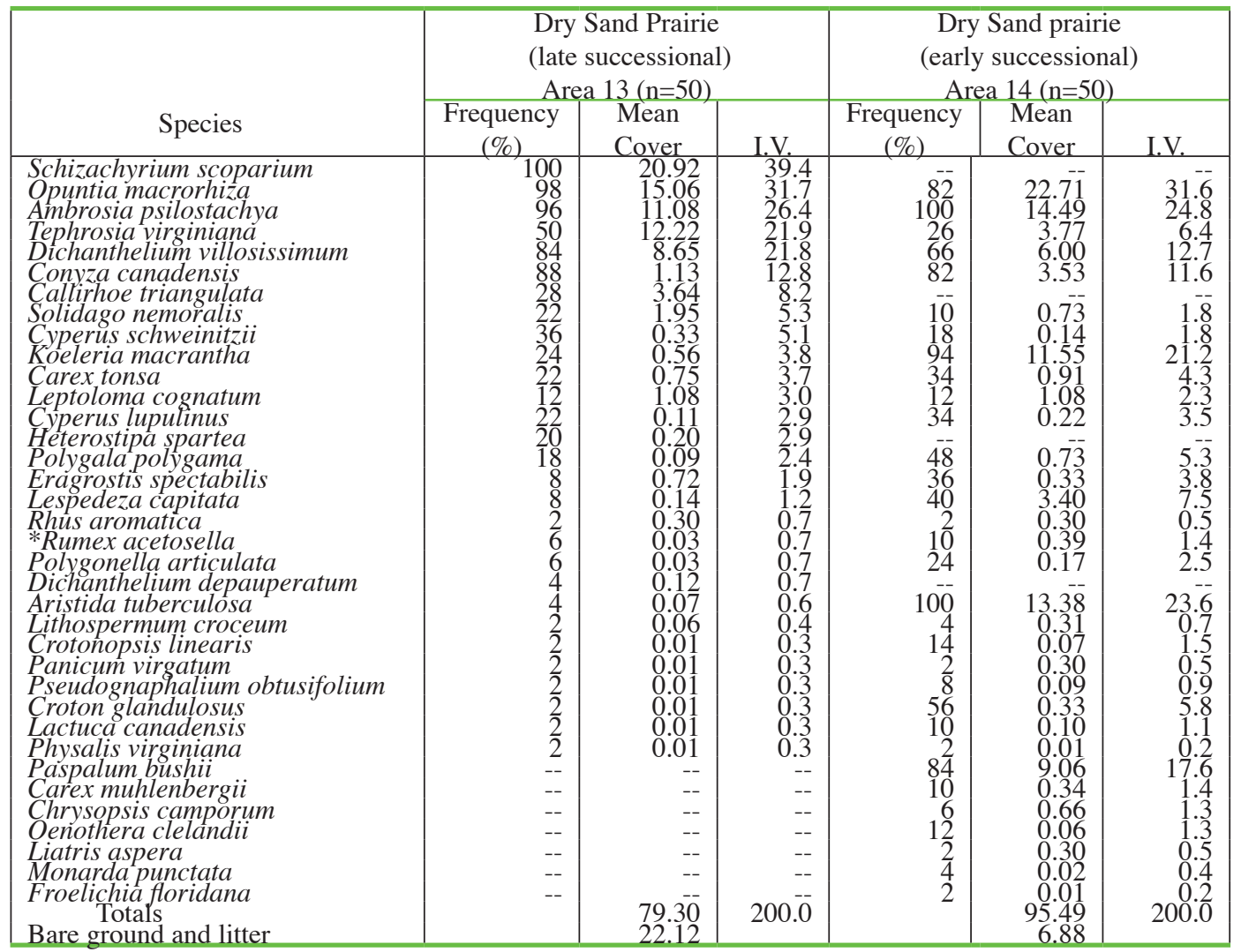

the other study areas with a ISs of $36.9 \%$ with the blowout community at Lost Mound to a high of 59.5 with the Schizachyrium/Poa community at Lost Mound (Table 14). This area is nearly $120 \mathrm{~km}$ south of the other study areas (Fig. 1).

A PCA biplot explained $44.5 \%$ of the variance in the first two axes, and with $66 \%$ of the variance explained in four axes. The ordination biplot indicated sample data are widely scattered in ordination space; however, three groups can be discerned (Fig. 2). Discriminating the sample data into three groupings was supported by results from cluster analysis. One grouping includes transects from all sites included in the study (LM 3, LM 7, LM 8, Ay10, TF13, and BR15) and is positively correlated with Schizachyrium scoparium, the dominant bunch grass in the sample transects and the species explaining the most variance on the first ordination axis. Associated species included Ambrosia psilostachya, Eragrostis spectabilis, Lespedeza capitata, Monarda punctata, Opuntia macrorhiza, Polygala polygama, and Solidago nemoralis. Another grouping comprised of transects from all sites except Big River (LM1, LM2, Ay11, Ay12, and TF14) is positively associated with species of blowouts and open sand habitats including Hudsonia tomensosa, Panicum virgatum, Koeleria macrantha, Cyperus grayoides, Croton glandulosa, Polygonum articulata, and Aristida tuberculosa. A third grouping is comprised solely of transects from Lost Mound (LM4, LM5, LM6, and LM9). Transects from this grouping are similar in that $S$. scoparium was missing or had 
Table 11. Frequency (\%), mean cover (\% of total cover), and importance value (I.V.) of ground layer species encountered in 2005 in a dry sand prairie community at Big River Natural Area, Henderson County, Illinois. (*non-native species)

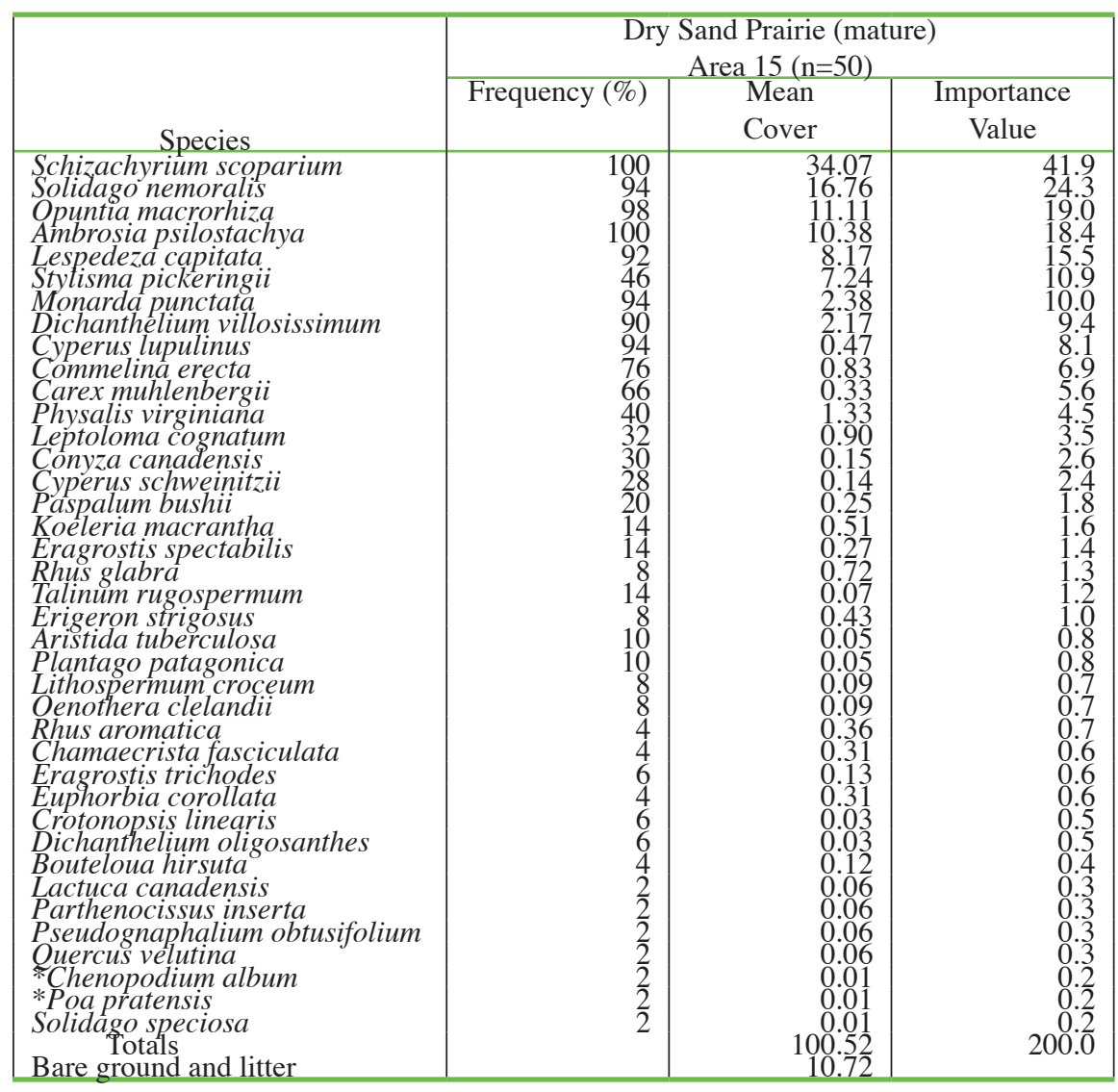


an IV less than $10 \%$. However, these transects grouped differently depending on choices of distance measure and linkage method in cluster analysis, indicating they were only nominally similar. Many adventive species were present in this third grouping including Achillea millefolium, Bromus inermis, Poa pratensis, Potentilla recta, and Rumex acetosella. Also, a few native grass species that are not bunch forming are associated with these transects including Sporobolus clandestinus, S. cryptandrus, Heterotheca spartea, and Triplasis purpurea.

The ecological meaning in the ordination axes is unclear. Neither of the species scores on the first two axes are correlated with the perceived conservatism of species (coefficients of conservatism) or wetness coefficients. A multiple regression of site characteristics (parameters of sand prairie community: species density, species richness, adventive species richness, mean coefficient of conservatism, and percent bare ground) onto the first two ordination axes explained $30.5 \%$ of the variance in the species data and $66.8 \%$ of the variance in the fitted species data. Results from forward selection of these site characteristics indicated that only one, percent bare ground, explained a significant amount of the variation $(P=0.01$, F-statistic 2.15). A triplot of species, sites, and site characteristics (not shown) indicated that percent bare ground was inversely associated with transects from Group 1 and positively associated with transects from Group 2.

\section{DISCUSSION}

Historical Summary: Historical information on the sand deposits of northwestern Illinois comes from the work of Gleason in 1908 (Gleason 1910). This study was completed nine years before the establishment of the Savanna Army Deport in 1918. Most of the information in that study consisted of detailed species lists with only a small amount of qualitative descriptive information on a few of the more common associations. The annotated lists of the species encountered, as well as the species he found in each association, give some indication of the complexity of this extensive sand prairie. As Dr. Gleason was at Lost Mound for only three short visits during 1908 (31 May-3 June, 12-24 June, 15-18 August) he made no attempt to ensure that a complete collection or a complete list of this sand region was developed, and many unusual locations for species were omitted (Gleason 1910).

Gleason (1910) described many of the plant associations and the successional processes that occur in the sand deposits throughout Illinois. His description of the Blowout Formation, its associations, and its succession to the Bunch-Grass Association are an excellent analysis of the complex and varied successional process in the sand deposits. He also described in detail the Mixed Consocies of the Bunch-Grass Association, which corresponds to the dry sand prairie community of White and Madany (1978). As described by Gleason (1910), this association was dominated by up to nine native bunch (clump) grasses and sedges, all common taxa of the sand deposits. Since the bunch grasses virtually excluded other growth beneath them, the remaining species of this association were restricted to the small areas of bare sand between the bunches. Gleason (1910) divided these secondary species into four ecological groups based on their habits and structure: large perennials and shrubs (that could compete with the bunch grasses); mat-plants (Selaginella rupestris, Opuntia macrorhiza); interstitials (mostly annuals with slender, frequently unbranched stems that were restricted to sand between the bunch grasses); and parasites (Orobanche fasciculata).

Since the early work of Gleason (1910), a few additional studies have been completed on the floristic composition and structure of the sand deposits of northwestern Illinois. In 1976 the Illinois Natural Areas Inventory (INAI) examined some of the sand prairies of this region (White 1978). During these studies frequency data were collected from 20 to 30 circular 0.25 $\mathrm{m}^{2}$ plots located along transects. None of these data were published but the results are available from Illinois Department of Natural Resources, Springfield, Illinois. Bowles et al. (2003) used many of these INAI sites in their study concerning the use of fire in the management of sand prairie vegetation.

Bunch-Grass Association of Gleason: Gleason (1910) reported that the Mixed Consocies of the Bunch-Grass Association dominated the sand deposits of Illinois, including the Hanover area of northwestern Illinois and the Oquawka area in Henderson County. Common bunch grasses were Koeleria macrantha, Leptoloma cognatum, and Schizachyrium scoparium 
Table 12. Size class density (\#/ha), basal area $\left(\mathrm{m}^{2} /\right.$ ha), relative values, importance value (I.V.), and average diameter $(\mathrm{cm})$ of the woody species encountered in 2005 in a degraded dry sand savanna community remnant at Big River Natural Area, Henderson County, Illinois. (* non-native species)

\begin{tabular}{|c|c|c|c|c|c|c|c|c|c|}
\hline Species & $\begin{array}{l}\text { Seed- } \\
\text { lings }\end{array}$ & $\begin{array}{l}\text { Small } \\
\text { Sap- } \\
\text { lings }\end{array}$ & $\begin{array}{l}\text { Large } \\
\text { Sap- } \\
\text { lings }\end{array}$ & $\begin{array}{l}\text { Trees } \\
\text { (\#/ha) }\end{array}$ & $\begin{array}{l}\text { Basal } \\
\text { Area } \\
\left(\mathrm{m}^{2} / \mathrm{ha}\right)\end{array}$ & $\begin{array}{l}\text { Rel. } \\
\text { Den. }\end{array}$ & $\begin{array}{l}\text { Rel. } \\
\text { Dom. }\end{array}$ & I. V. & $\begin{array}{l}\text { Av. } \\
\text { Diam. } \\
(\mathrm{cm})\end{array}$ \\
\hline Quercus velutina & 7500 & 1594 & 106 & 298 & 9.386 & 50.8 & 54.2 & 105.0 & 18.7 \\
\hline Ouercus marilandica & 1563 & 375 & 131 & 286 & $\begin{array}{r}7.880 \\
.034\end{array}$ & 48.6 & 45.6 & 94.2 & 7.3 \\
\hline $\begin{array}{l}\text { Dniperus virginiana } \\
\text { Prunus serotina }\end{array}$ & 938 & 406 & 75 & 2 & 0.24 & 0.3 & 0.1 & 0.4 & 12.3 \\
\hline Rubus allegheniensis & 3281 & 750 & & & & & & & \\
\hline Rubus occidentalis & 2031 & 1531 & -- & -- & -- & -- & -- & -- & -- \\
\hline Cornus drummondii & 1563 & 4250 & -- & - & -- & -- & $\begin{array}{l}-- \\
--\end{array}$ & -- & -- \\
\hline Ribes missouriense & 781 & 438 & -- & -- & -- & -- & -- & -- & -- \\
\hline Rhus aromatica & 313 & 656 & -- & -- & -- & -- & -- & -- & -- \\
\hline Rhus glabra & 156 & 688 & -- & -- & -- & -- & -- & -- & -- \\
\hline * Elaeagnus umbellata & . & & . & 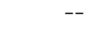 & -- & -- & . & -- & -- \\
\hline $\begin{array}{l}\text { Gleditsia triacanthos } \\
\text { *Morus alba }\end{array}$ & $\begin{array}{l}-- \\
--\end{array}$ & 31 & $\begin{array}{l}-- \\
--\end{array}$ & $\begin{array}{l}-- \\
--\end{array}$ & $\begin{array}{l}-- \\
--\end{array}$ & $\begin{array}{l}-- \\
--\end{array}$ & $\begin{array}{l}-- \\
--\end{array}$ & $\begin{array}{l}-- \\
--\end{array}$ & $\begin{array}{l}-- \\
--\end{array}$ \\
\hline * Rosa multiflora & $1937 \overline{6}$ & $\begin{array}{r}31 \\
11187\end{array}$ & 318 & 588 & 17324 & 1000 & 1000 & 2000 & \\
\hline
\end{tabular}

though all of the other graminoid taxa were also encountered, but rarely dominant. Overall, Gleason (1910) found that these three bunch grasses were "so regularly present and so frequently associated with each other that they may be regarded as the most typical grasses of the consocies." Except on rare occasions where one or two of the bunch-grass species dominated a small area, the remaining grasses never occupied large portions of the ground space. Essentially all of the other species reported by Gleason (1910) for northwestern Illinois were found during the present study.

Patterns of bunch grasses diversity and abundance involve many factors and are scale and habitat dependent. All of the common bunch grasses of northern Illinois occur throughout these sand deposits, their presence in any particular area related to disturbance, moisture, and many other biotic and abiotic factors. Within the sand deposits of northwestern Illinois, the high-quality areas surveyed were mostly associated with the bunch grass Schizachyrium scoparium. This species was generally the dominant or subdominant species of these high-quality sites (Fig. 2). Also, these high-quality areas were negatively associated with exotic, non-native species. In contrast, areas of disturbance where blowing sand was common were positively associated with nonnative species. The bunch grasses Dichanthelium villosissimum, Koeleria macrantha, and Panicum virgatum were positively associated with these areas of blowing sand (Fig. 2). In mid-successional areas, where many native sand prairie species were common, the concen- tration of non-native species was highly variable, and many of the grasses present were not well-developed bunch grasses. Here Dichanthelium oligosanthes, D. perlongum, Heterostipa spartea, Sporobolus clandestinus, S. crypandrus, Triplasis purpurea, and the non-native Poa pratenisis were common. The mid-summer bunch grass Leptoloma congnatum was positively associated with these mid-successional sites (Fig. 2).

Typical of the bunch-grass association, areas of bare ground and litter usually exist between the clumps. Generally the clumps of Schizachyrium scoparium were $15-40 \mathrm{~cm}$ across, nearly circular in outline, and formed dense masses. Some of the larger clumps of this species had dead centers forming rings in which no other species were observed. Most of the other common grasses of this bunchgrass association, particularly Dichanthelium villosissimum and Koeleria macrantha, had similar growth forms, but formed much smaller clumps. During the present study the mean cover of bare ground and litter in mature dry sand prairies was between $10 \%$ and $38 \%$, in successional dry sand prairies between $6 \%$ and $30 \%$, and in blowouts and blowing sand communities between $41 \%$ and $63 \%$. Mature dry sand prairie communities in the Illinois River sand deposits of central Illinois also had extensive areas of open sand. At Long Branch Nature Preserve bare ground ranged from $38 \%$ to $44 \%$ in a mature dry sand prairie, while in a disturbed sand community bare ground averaged 59\% (Phillippe et al. 2004). In another Mason County sand prairie complex at Henry 
Allan Gleason Nature Preserve, bare ground and litter averaged $35 \%$ in a mature dry sand prairie, $47 \%$ to $52 \%$ in two successional communities, and $83 \%$ in a blowout community (McClain et al. 2004).

Between the clumps of grasses other graminoid species were common along with many prairie forbs. Though these sand prairies are part of the tallgrass prairie region, the species composition and relative abundance of the species in the interstitial areas between bunch grasses usually differ from those found in tallgrass prairies. Soil moisture retention of the sandy soil is low, and sand prairies generally support species that tolerate drier conditions. Also, productivity is generally low in sand prairies, due mostly to low soil organic matter content and low available nitrogen (Anderson et al. 1994). Very few native legumes were found in the communities studied. At Lost Mound, Tephrosia virginiana was the only native legume commonly encountered in the plots, other native legumes being rare. In contrast, at Ayers, Thomson/Fulton and Big River, both Tephrosia virginiana and Lespedeza capitata (roundheaded bush clover) were relatively common, generally being among the top 10 species in IV.

Other Illinois Sand Deposits: Sand prairie remnants have also been studied in the Green River Lowland Section of the Grand Prairie Natural Division in northwestern Illinois. These remnants are between 50 and $75 \mathrm{~km}$ east of the Mississippi River, are adjacent to the Mississippi River sand deposits, and were deposited during warm periods near the end of Wisconsian Glaciation. One sand prairie remnant is on a shallow ridge surrounded by wet sand prairies and sedge meadows at the Richardson Wildlife Foundation (Handel et al. 2003). Here Sorghastrum nutans and Schizachyrium scoparium were dominant species, while the important forbs included Euthamia graminifolia, Solidago nemoralis, and Liatris aspera. The second sand prairie is associated with a dune ridge at Foley Sand Prairie Nature Preserve (McClain et al. 2003). Though Schizachyrium scoparium dominated this site, the remainder of the flora indicated more mesic conditions. On Foley sand prairie Opuntia macrorhiza was not encountered, Dichanthelium villosissimum was rare, and Ambrosia psilostachya was eighth in IV. Both prairie remnants were wetter than the sand prairies encountered in the Mississippi River sand deposits. Continued on page 216

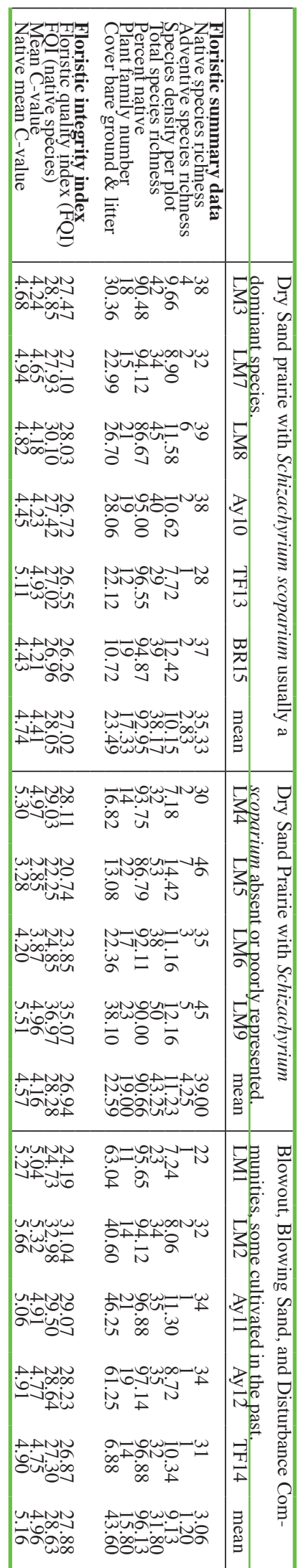




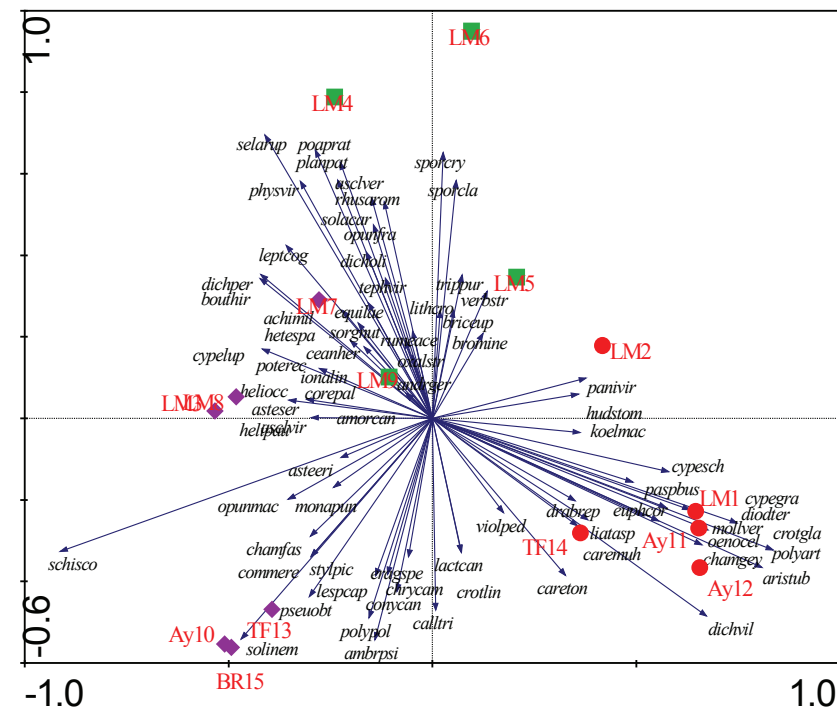

Figure 2. Biplot of species scores and plot loadings of the first two axes of a Principal Components Analysis (PCA) showing ground cover data, using importance values from 15 transects $(n=$ 50 plots/transect) at Lost Mound (LM), Ayers Sand Prairie Nature Preserve (Ay), Thomson-Fulton Nature Preserve (TF), and Big River Natural Area (BR). Uncommon species were deleted.

achimil $=$ Achillea millefolium ambrpsi = Ambrosia psilostachya amorcan = Amorpha canescens andoger $=$ Andropogon gerardii aristub $=$ Aristida tuberculosa asclver $=$ Asclepias verticillata asclvir = Asclepias viridiflora asteeri $=$ Aster ericoides asteser $=$ Aster sericeus bouthir = Bouteloua hirsuta briceup = Brickellia eupatorioides bromine $=$ Bromus inermis calltri $=$ Callirhoe triangulata caremuh $=$ Carex muhlenbergii careton $=$ Carex tonsa ceanher $=$ Ceanothus herbaceus chamgey $=$ Chamaesyce geyer $i$ chamfas = Chamaecrista fasciculata chrycam $=$ Chrysopsis camporum commere $=$ Commelina erecta conycan = Conyza canadensis corepal $=$ Coreopsis palmata crotgla $=$ Croton glandulosus crotlin $=$ Crotonopsis linearis cypegra $=$ Cyperus grayoides cypelup $=$ Cyperus lupulinus cypesch $=$ Cyperus schweinitzii dicholi $=$ Dichanthelium oligosanthes dichper $=$ Dichanthelium perlongum dichvil $=$ Dichanthelium villosissimum diodter $=$ Diodia teres drarep $=$ Draba reptans equilae = Equisetum laevigatum euphcor = Euphorbia corollata eragspe $=$ Eragrostis spectabilis heliocc $=$ Helianthus occidentalis helipau $=$ Helianthus pauciflorus hetespa $=$ Heterostipa spartea hudstom = Hudsonia tomentosa ionalin = Ionactis linariifolius koelmac $=$ Koeleria macrantha lactcan = Lactuca canadensis leptcog = Leptoloma cognatum lespcap = Lespedeza capitata liatasp = Liatris aspera litocro = Lithospermum croceum mollver $=$ Mollugo verticillata monapun = Monarda punctata oenocel $=$ Oenothera clelandii opunfra $=$ Opuntia fragilis opunmac $=$ Opuntia macrorhiza oxalstr $=$ Oxalis stricta panivir = Panicum virgatum paspbus $=$ Paspalum bushii physvir = Physalis virginiana planpat $=$ Plantago patagonica poaprat $=$ Poa pratensis polyart $=$ Polygonella articulata polypol = Polygala polygama poterec $=$ Potentilla recta pseuobt $=$ Pseudognaphalium obtusifolium rhusaro $=$ Rhus aromatica rumeace $=$ Rumex acetosella schisco = Schizachyrium scoparium selarup $=$ Selaginella rupestris solacar = Solanum carolinense solinem $=$ Solidago nemoralis sorgnut $=$ Sorghastrum nutans sporcla $=$ Sporobolus clandestinus sporcry $=$ Sporobolus cryptandrus stylpic $=$ Stylisma pickeringii tephvir = Tephrosia virginiana trippur $=$ Triplasis purpurea verbstr = Verbena stricta violped $=$ Viola pedata 


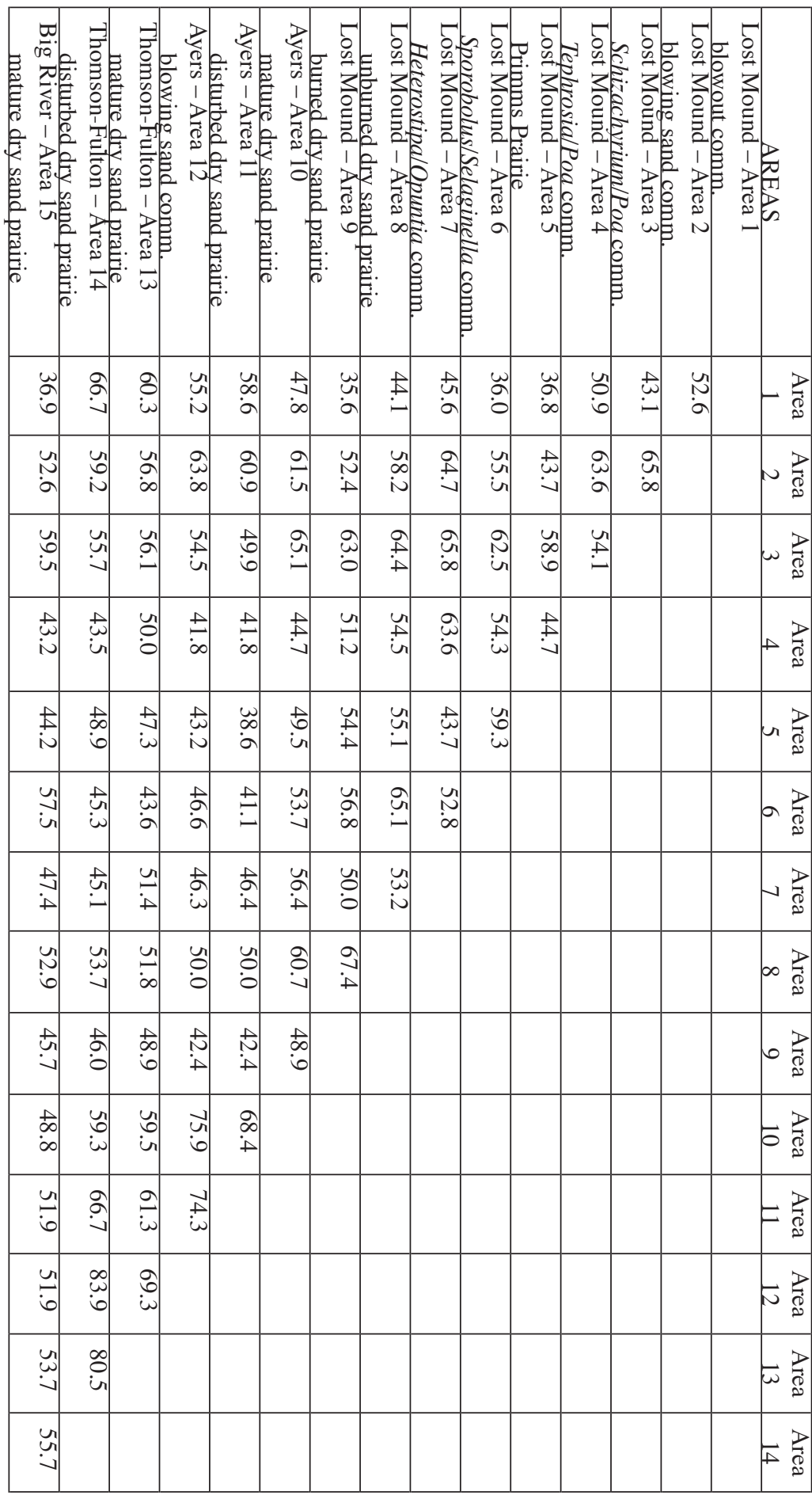


The flora of the relatively mature dry sand prairie studied in the Mississippi River sand deposits is very similar to that of sand prairies associated with the Illinois River sand deposits in central Illinois. Dry sand prairies at Henry Allan Gleason Nature Preserve (McClain et al. 2004) and Long Branch Nature Preserve (Phillippe et al. 2004), both in Mason County, have nearly identical dominant species as those in northwestern Illinois. Both of these Mason County prairies were dominated by Schizachyrium scoparium while Opuntia humifusa, Dichanthelium villosissimum, and Ambrosia psilostachya were among the top six species in IV. Many subordinate species of these three dry sand prairies are also identical. In the mature dry sand prairies examined during the present study, many of the same species were high in IV. In many of the successional and disturbed communities examined during the present study, Schizachyrium scoparium was rarely encountered, though it was abundant throughout surrounding areas. The low incidence of this species may be related to its association with vesicular arbuscular mycorrhizal fungi that, for some reason, may not be present in the soil or may be due to the lack of certain soil nutrients (Dhillion et al. 1992, Anderson and Liberta 1992).

Management Implications: To study longterm changes in burned and unburned sand prairie remnants, many of the sites listed in the INAI were surveyed by Bowles et al. (2003) in 1996 and the results compared with the data obtained in the original INAI surveys. They studied seven sites: three that were managed with fire over the 20-year period and four that were not. Overall, native species richness per plot increased only on burned sites, whereas alien species richness per plot increased only on unburned sites. In the unburned sand prairies there was an increase in the alien grasses Bromus inermis and Poa pratensis that was accompanied by a decline in the native Schizachyrium scoparium, Heterostipa spartea, Echinacea pallida, Helianthus pauciflorus, and Coreopsis palmata (Bowles et al. 2003).

Similar results were observed during the present study, particularly at Lost Mound. Here fire suppression has been the rule since the army obtained the area in 1918 and adventive, cool-season grasses were planted into areas of the prairie. Fire suppression has undoubtedly resulted in adventive species becoming important components of this dry sand prairie, particularly in high disturbance areas and successional communities. Presently fire is occasionally used in many of the nature preserves in the Mississippi River sand deposits, and at Lost Mound a burning program is being initiated since the land was transferred to the U.S. Fish and Wildlife Service in 2003 (Nÿboer, personal observations). It is generally accepted that the establishment of prairie species is stimulated by fire and the removal of litter. Fires, as well as patch disturbances, generally increase species richness, particularly native prairie forbs that mostly occur as interstitial species in bunch-grass communities (Bowles et al. 2003). All available information indicates that fire, particularly early spring fires, are important in decreasing the extent of the cool-season, Eurasian grasses, decreasing the density and cover of adventive species, and increasing the density and cover of native sand prairie species.

Adventive Species: Presently adventive species are more abundant at Lost Mound than at the other natural areas examined. Adventive species are commonly associated with disturbances, particularly ground disturbances associated with human activity, such as roads, buildings, and agriculture, as well as overgrazing. At Lost Mound more than 100 adventive species were found associated with the prairie, mostly in areas of major disturbances. Within the plant communities studied at Lost Mound, adventive taxa were sometimes abundant. Poa pratensis was the most common adventive species in the study areas, being very abundant in the study plots in areas that had been heavily grazed in the past. Another commonly observed adventive grass was Bromus inermis, while Rumex acetosella and Potentilla recta were sometimes common in the study plots. At Ayers, Thomson/Fulton, and Big River, adventive species were less common. At these three sites Poa pratensis had an IV of 4.1 or lower within the plots. The only other adventive species encountered were Achillea millefolium, Bromus tectorum, Chenopodium album, Mollugo verticillata, and Rumex acetosella. Overall, in all of the natural areas examined, adventive species accounted for about $20 \%$ of the flora (Appendix I). 
Few adventive legumes were recorded for the study plots. At Lost Mound, however, many were found in heavily disturbed areas, particularly along roadsides and in areas where cattle concentrations had been high (Appendix I). The most common adventive legume observed at Lost Mound was Securigera varia (crown vetch). Symstad (2004) found that the presence of crown vetch significantly increased soil nitrogen availability and significantly decreased native species richness and cover. High nitrogen levels caused a dramatic increase in $\mathrm{Poa}$ pratensis cover, which could hinder restoration efforts by competition with native species.

\section{Forest and Savanna Communities: Gleason}

(1910) described the Lost Mound area as:

"The sand deposits are chiefly prairie, but a belt of forest lies along the river, and tongues and irregular areas of forest project out into the prairie, in some places extending nearly across." Presently timber harvesting, grazing, oak wilt disease, and fire suppression have heavily modified the forest and savanna communities. A narrow belt of timber still exists along the river adjacent to Lost Mound. Quercus velutina dominates the dunes just back from the river, while a floodplain forest, dominated by Acer saccharinum, occurs on the frontal flats and the deposition area behind the navigation dam that crosses the Mississippi River near the north end of Lost Mound. On the sandy terrace behind the riverside dunes, prairie dominates. In this prairie scattered degraded savanna communities occur that are dominated by $Q$. velutina. In these sand forests and savannas, overstory species diversity is relatively low with $Q$. velutina the dominant species on dry sites and Q. alba becoming an important component of moister sites. At Big River State Forest, Q. marilandica becomes an important overstory component in the dry sand forest.

Throughout the sand deposits of Illinois, Quercus velutina generally dominates with none or only a few other Quercus species and sometimes with a few species of the genus Carya (hickories). In the Kankakee River sand deposits dry to dry-mesic sand savanna and forests communities are dominated by $Q$. velutina, which accounted for $75 \%$ to $97 \%$ of the IV. On more mesic sites $Q$. alba was the only other tree species commonly encountered (Johnson and Ebinger 1992). In the Illinois River sand deposits, in contrast, overstory species diversity is sometimes higher. Again $Q$. velutina is the dominant species, but $Q$. marilandica is usually well established along with occasional individuals of Carya texana (black hickory) and C. tomentosa (mockernut hickory) (McClain et al.2002). The forest communities in the Big River State Forest, though heavily degraded by fire suppression, are similar to the dry sand forests of the Illinois River sand deposits. 


\section{LITERATURE CITED}

Anderson, R.C., and A.E. Liberta. 1992. Influence of supplemental inorganic nutrients on growth, survivorship, and mycorrhizal relationships of Schizachyrium scoparium (Poaceae) grown in fumigated and unfumigated soil. American Journal of Botany 79:406-414.

Anderson, R.C., B.A.D. Hetrick, and G.W.T. Wilson. 1994. Mycorrhizal dependence of Andropogon gerardii and Schizachyrium scoparium in two prairie soils. American Midland Naturalist 132:366-376.

Bailey, A.W., and C.E. Poulton. 1968. Plant communities and environmental relationships in a portion of the Tillamook burn, northwestern Oregon. Ecology 49:1-13.

Bowles, M., and M. Jones. 1995. Management and research needs for endangered sand threatened plants, sand prairie vegetation and habitat-restricted animal species at the Savanna Army Depot, Carroll County and Jo Daviess County, Illinois. Morton Arboretum, Lisle, Illinois. $21 \mathrm{pp}$. Unpublished report submitted to the Savanna Army Depot.

Bowles, M.L., M.D. Jones, and J.L. McBride. 2003. Twenty-year changes in burned and unburned sand prairie remnants in northwestern Illinois and implications for management. American Midland Naturalist 149:35-45.

Coates, D.T., S.E. Jenkins, and J.E. Ebinger. 1992. Woody vegetation survey of Barkhausen Woods, a closed canopy sand forest in Mason County, Illinois. Erigenia 12:1-6.

Daubenmire, R. 1959. A canopy coverage method of vegetation analysis. Northwest Science 33:43-64.

Dhillion, S.S., R.C. Anderson, and A.E. Liberta. 1992. Effects of fire on the mycorrhizal ecology of little bluestem (Schizachyrium scoparium). Canadian Journal of Botany 66:706-713.

Feist, M.A., M.J. Morris, L.R. Phillippe, J.E. Ebinger, and W.E. McClain 2006. Wet sand prairie communities of Matanzas Nature Preserve, Mason County, Illinois. (In review).
Gleason, H.A. 1910. The vegetation of the inland sand deposits of Illinois. Bulletin of the Illinois State Laboratory of Natural History 9:21-174.

Gleason, H.A., and A. Cronquist. 1991. Manual of the vascular flora of northeastern United States and adjacent Canada. Second Edition. The New York Botanical Garden, The Bronx. $1 \mathrm{xxv}+910 \mathrm{pp}$.

Handel, W.C., L.R. Phillippe, and J.E. Ebinger. 2003. Floristic assessment of sand prairies and sedge meadows, Lee County, Illinois. The Prairie Naturalist 35:33-46.

Hart, C.A., and H.A. Gleason 1907. On the biology of the sand areas of Illinois. Bulletin of the Illinois State Laboratory of Natural History 7:137-272.

Herkert, J.R., and J.E. Ebinger 2002. Editors. Endangered and threatened species of Illinois: status and distribution. Volume 1: plants. Endangered Species Protection Board, Springfield. $161 \mathrm{pp}$.

Jenkins, S.E., J.E. Ebinger, and W.E. McClain. 1991. Woody vegetation survey of Bishop's Woods, a sand forest in Mason County, Illinois. Transactions of the Illinois State Academy of Science 84:20-27.

Johnson, K.C., and J.E. Ebinger. 1992. Effects of prescribed burns on the woody vegetation of a dry sand savanna, Hooper Branch Nature Preserve, Iroquois County, Illinois. Transactions of the Illinois State Academy of Science 85:105-111.

Johnson, K.C., and J.E. Ebinger. 1995. Effects of different fire regimes on the ground layer vegetation of a dry sand savanna, Hooper Branch Nature Preserve, Iroquois County, Illinois. Erigenia 14:37-40.

King, J.E. 1981. Late Quaternary vegetational history of Illinois. Ecological Monographs 51:43-62.

McClain, W.E., R.D. McClain, and J.E. Ebinger. 1997. Flora of temporary sand ponds in Cass and Mason counties, Illinois. Castanea 65-73. 
McClain, W.E., L.R. Phillippe, and J.E. Ebinger. 2003. Floristic assessment of Foley Sand Prairie Nature Preserve, Lee County, Illinois. Transactions of the Illinois State Academy of Science 96:255-263.

McClain, W.E., L.R. Phillippe, and J.E. Ebinger. 2004. Floristic assessment of the Henry Allan Gleason Nature Preserve, Mason County, Illinois. Castanea 70:146-154.

McClain, W.E., S.D. Turner, and J.E. Ebinger. 2002. Vegetation of forest communities at the Sand Prairie-Scrub Oak Nature Preserve, Mason County, Illinois. Transactions of the Illinois State Academy of Science 95:37-46.

McCune, B., and M.J. Mefford. 1999. PCORD. Multivariate analysis of ecological data, Version 4. MjM Software Design, Gleneden Beach, Oregon.

McDowell, B., J. Newman, and J. Ebinger. 1983. Survey of the woody vegetation of the Kankakee Sand Area Section of Indiana and Illinois. Proceedings of the Indiana Academy of Science 93:187-193.

McIntosh, R.P. 1957. The York Woods. A case history of forest succession in southern Wisconsin. Ecology 38:29-37.

Midwestern Regional Climate Center. 2005. http://mcc.sws.uiuc.edu

Mohlenbrock, R.H. 2002. Vascular flora of Illinois. Southern Illinois University Press, Carbondale and Edwardsville. $\mathrm{x}+490 \mathrm{pp}$.

Mueller-Dombois, D., and H. Ellenberg. 1974. Aims and methods of vegetation ecology. John Wiley and Sons, New York. 547 pp.

Phillippe, L.R., M.A. Feist, and J.E. Ebinger. 2004. Vascular flora of Long Branch Nature Preserve, Mason County, Illinois. Transactions of the Illinois State Academy of Science 97:197-208.

Ray, B.W., R. Rehner, and J.B. Fehrenbacher. 1975. Soil survey: Carroll County, Illinois. University of Illinois Agricultural Experiment Stations in cooperation with Soil Conservation Service, U.S. Department of Agriculture, Urbana. 138 pp. +39 maps.
Robertson, K.R., L.R. Phillippe, G.A. Levin, and M.J. Moore. 1997. Delineation of natural communities, checklist of vascular plants, and new locations for rare pants at the Savanna Army Depot, Carroll and Jo Daviess counties. Illinois Natural History Survey, Center for Biodiversity Technical Report 1997 (2). Unpublished report prepared for the Illinois Department of Natural Resources, Division of Natural Heritage, Springfield. 90 pp. + map.

Sabata, L.R. 1995. Soil survey of Whiteside County, Illinois. United States Department of Agriculture, Soil Conservation Service, in cooperation with the Illinois Agricultural Experiment Station, Champaign. ix +300 pp. + 103 maps.

Schwegman, J.E. 1973. Comprehensive plan for the Illinois Nature Preserves System. Part 2. The natural divisions of Illinois. Illinois Nature Preserves Commission, Rockford. 32 pp. + map.

Smith, R.S., O.I. Ellis, E.E. DeTurk, F.C. Bauer, and L.H. Smith. 1928. Whiteside County soils. University of Illinois Agricultural Experiment Station. Soil Report 40:1-65.

Symstad, A.J. 2004. Secondary invasion following the reduction of Coronilla varia (crownvetch) in sand prairie. American Midland Naturalist 152:183-189.

Taft, J.B., C. Hauser, and K.R. Robertson. 2006. Estimating floristic integrity in tallgrass prairie. Biological Conservation 131:42-51.

Taft, J.B., G.S. Wilhelm, D.M. Ladd, and L.A. Masters. 1997. Floristic quality assessment for vegetation in Illinois, a method for assessing vegetation integrity. Erigenia 15:1-95.

Tegeler, R.A. 1996. Soil survey of Jo Daviess County. United States Department of Agriculture, Natural Resources Conservation Service, in cooperation with the Illinois Agricultural Experiment Station, Champaign. vii +224 pp. + 91 maps. 
Ter Braak, C.J.F. 1995. Ordination. Pages 91-173 in R.H.G. Jongman, C.J.F Braak, and O.R.F Van Tongeren, eds. Data analysis in community and landscape ecology. Cambridge University Press, New York, New York.

Ter Braak, C.J.F., and I.C. Prentice. 1988. A theory of gradient analysis. Advances in Ecological Research 18:271-317.

Veale, P.T., and H.L. Wascher. 1956. Henderson County soils. Soil Report 77. University of Illinois Agricultural Experiment Station, Urbana. 64 pp. + 45 maps.

Vestal, A.G. 1913. An associational study of Illinois sand prairie. Bulletin of the Illinois State Laboratory of Natural History 10:1-96.

White, J. 1978. Illinois natural areas inventory. Technical report. Volume I. Survey methods and results. Illinois Natural Areas Inventory, Urbana. xix +426 pp.

White, J., and M.H. Madany. 1978. Classification of natural communities in Illinois. Pages 310-405 in Illinois natural areas inventory. Technical report. (J. White, ed). Illinois Natural Areas Inventory, Urbana.

Willman, H.B., and J.C. Frye. 1970. Pleistocene stratigraphy of Illinois. Illinois State Geological Survey Bulletin 94:1-204. 


\section{APPENDIX I}

Vascular plant species found in the Lost Mound Unit Savanna District of the Upper Mississippi River National Wildlife and Fish Refuge, Ayers Sand Prairie Nature Preserve, Thomson-Fulton Nature Preserve, and Big River State Forest, are listed alphabetically by family under major plant groups. An asterisk indicates non-native species. Collecting numbers preceded by $\mathrm{G}$ were collected by Henry A. Gleason and are deposited in the University of Illinois Herbarium, Urbana, Illinois (ILL). Collecting numbers proceeded by P or S were collected by Loy R. Phillippe or Amy Symstad and are deposited in the Illinois Natural History Survey Herbarium, Champaign, Illinois (ILLS). Collecting numbers preceded by E were collected by John E. Ebinger and are deposited in the Eastern Illinois University Herbarium, Charleston, Illinois (EIU). In addition, a few of the species were observed but not collected, and a few were reported by the Illinois Natural Areas Inventory (INAI) for which we could not find vouchers. The letter after each collecting number indicates the collecting site: $\mathrm{s}=$ Lost Mound; $\mathrm{a}=$ Ayers Sand Prairie Nature Preserve; $\mathrm{t}=$ Thomson-Fulton Nature Preserve; $b=$ Big River State Forest.

\section{FERNS AND FERN-ALLIES}

\section{Aspleniaceae}

Asplenium platyneuron (L.) Oakes: P27959s

\section{Dennstaedtiaceae}

Pteridium aquilinum (L.) Kuhn: P28006s

\section{Dryopteridaceae}

Athyrium filix-femina (L.) Martens ssp. angustum (Willd.) R.T. Clausen: P27491s

Cystopteris protrusa (Weatherby) Blasdell: P27794s

Dryopteris carthusiana (Villars) H.P. Fuchs: P27487s

Woodsia obtusa (Spreng.) Torr.: P28578s

\section{Equisetaceae}

Equisetum arvense L.: P27198s

Equisetum x ferrissii Clute: P28587s

Equisetum hyemale L.: P27812s

Equisetum laevigatum A. Br.: P27529s; P36240a; E30652t

Equisetum pratense Ehrh.: P27195s

\section{Onocleaceae}

Onoclea sensibilis L.: P28270s

\section{Ophioglossaceae}

Botrychium dissectum Spreng.: P28441s

Botrychium virginianum (L.) Sw.: P27483s

Ophioglossum pusillum Raf.: P28440s

\section{Osmundaceae}

Osmunda claytoniana L.: P28449s

\section{Pteridaceae}

Adiantum pedatum L.: P27485s

\section{Selaginellaceae}

Selaginella rupestris (L.) Spring.: P27158s; E30611a; E31566t 


\title{
GYMNOSPERMS
}

\section{Cupressaceae}

Juniperus virginiana L.: P27503s; E31263a; E30599t; E31667b

\section{Pinaceae}

*Pinus banksiana Lamb.: P28581s; E30600t; E31952b

*Pinus resinosa Ait.: P28446s

*Pinus sylvestris L.: P37108a; E31231t

\section{MONOCOTS}

\section{Agavaceae}

*Yucca smalliana Fern.: E31567t

\section{Alismataceae}

Alisma subcordatum Raf.: P27974s

Sagittaria latifolia Willd.: M3411; P28238s

\author{
Araceae \\ Arisaema dracontium (L.) Schott: P27488s \\ Arisaema triphyllum (L.) Schott: P27201s
}

\section{Commelinaceae}

Commelina erecta L.: P27849s; INAIa; E31378t; E31467b

Tradescantia ohiensis Raf.: P27424s; E30628a; E30653t; E31699b

\section{Cyperaceae}

Bolboschoenus fluviatilis (Torr.) Sojak: P28569s

Bulbostylis capillaris (L.) C.B. Clarke: P27832s; P37248b

Carex bicknellii Britt.: P27408s; E30630a

Carex blanda Dewey: P27280s

Carex brachyglossa Mack.: P27518s

Carex brevior (Dewey) Mack.: P27420s

Carex cephalophora Muhl. ex Willd.: E31867b

Carex conjuncta Boott: P27434s

Carex cristatella Britt.: P27810s

Carex duriuscula C.A. Meyer: P27326s

Carex festucacea Schk.: E31376a; P36717t

Carex frankii Kunth: P27963s

Carex gravida L.H. Bailey: P27498s

Carex grayi Carey: P27453s

Carex grisea Wahl: P27452s

Carex hirtifolia Mack.: P27472s

Carex hystericina Muhl.: P27494s

Carex laeviconica Dewey: P27796s

Carex lupulina Willd.: P27552s

Carex meadii Dewey: P27209s

Carex molesta Mack.: P27517s

Carex muhlenbergii Schk.: P27425s; E30629a; E30654t; E31468b

Carex pensylvanica Lam.: P27211s; E31321a; E31311t; E31664b

Carex rosea Schk.: P27437s

Carex scoparia Schk.: P27438s 
Carex stipata Muhl.: P27471s

Carex stricta Lam.: P27430s

Carex tonsa (Fern.) Bickn.: P27159s; E31320a; E31379t

Carex tribuloides Vahl: P27551s

Carex typhina Michx.: P27792s

Carex vulpinioidea Michx.: P27756s

Cyperus erythrorhizos Muhl.: P28085s

Cyperus esculentus L.: P28084s

Cyperus grayoides Mohlenbr.: P27829s; P37104a; E31509t

Cyperus lupulinus (Spreng.) Marcks var. lupulinus: P27512s; P36211a; E31508t; E31865b

Cyperus lupulinus (Spreng,) Marcks var. macilentus (Fern.) Marcks: P27718s; E31864b

Cyperus $x$ mesochorus Geise: E31866b

Cyperus odoratus L.: P28044s

Cyperus schweinitzii Torr.: P27717s; E31709a; E31380t; E31469b

Cyperus squarrosus L.: P28226s

Eleocharis acicularis (L.) Roem. \& Schultes: P27543s

Eleocharis erythropoda Steud.: P28120s

Eleocharis ovata (Roth) Roem. \& Schultes var. obtusa (Willd.) Kukenth: P27975s

Scirpus atrovirens Willd.: P27783s

Scirpus cyperinus (L.) Kunth: P27981s

\section{Hydrocharitaceae}

Elodea nuttallii (Planch.) St. John: P28242s

Vallisneria americana Michx.: P28245s

\section{Iridaceae}

Iris shrevei Small: P27800s

Sisyrinchium albidunm Raf.: E31322a; E31470b

Sisyrinchium campestre Bickn.: P27300s; E31665b

Sisyrinchium mucronatum Michx.: E31323a

\section{Juncaceae}

Juncus interior Wieg.: P27782s

Juncus tenuis Willd.: P27757s

\section{Lemnaceae}

Lemna minor L.: P28095s

Spirodela polyrhiza (L.) Schleiden: P28264.1s

Wolffia columbiana Karst: P28264.2s

\section{Liliaceae}

Allium canadense L.: P27788s

*Asparagus officinalis L.: P27457s

*Hemerocallis fulva (L.) L.: P27815s

Polygonatum commutatum (Schult.) A. Dietr.: P27716s

Polygonatum biflorum (Walt.) Ell.: E31700b

Smilacina stellata (L.) Desf.: P27166s

\section{Najadaceae}

Najas minor All.: P27814s 


\section{Orchidaceae}

Galearis spectabilis (L.) Raf.: P27332s

Liparis liliifolia (L.) Rich.: P28439s

Spiranthes lacera (Raf.) Raf.: S364s

\section{Poaceae}

Agrostis gigantea Roth: P27761s

Agrostis hyemalis (Walt.) BSP.: P27509s; INAIa

Alopecurus carolinianus Walt.: P27440s

Andropogon gerardii Vitman: P28108s; E31264a; INAIt; E31954b

Aristida basiramea Engelm.: P328114s; P36235a

Aristida oligantha Michx.: P28112s

Aristida tuberculosa Nutt.: P28028s; E31265a; E31232t; P37242b

Bouteloua curtipendula (Michx.) Torr.: P27864s

Bouteloua gracilis (HBK.) Lag.: P28554s

Bouteloua hirsuta Lag.: P27940s; P36216a; INAIt; E31855b

*Bromus inermis Leyss.: P27499s; E30631a; E30656t; E31701b

Bromus kalmii Gray: P28101s

*Bromus racemosus L.: P27502s; E30655t

*Bromus tectorum L.: P27311s; E30612a; E30601t; E31856b

Calamovilfa longifolia (Hook.) Scribn.: P28424s; E31266a; E31233t; E31471b

Cenchrus longispinus (Hack.) Fern.: P27968s; INAIa; INAIt; E31857b

*Chloris verticillata Nutt.: P27732s

Cinna arundinacea L.: P28433s

*Dactylis glomerata L.: P27470s

Dichanthelium acuminatum (Sw.) Gould \& Clark var. fasciculatum (Torr.) Freckm.: P27713s

Dichanthelium acuminatum (Sw.) Gould \& Clark var. implicatum (Scribn.) Gould \& Clark: P28129s

Dichanthelium depauperatum (Muhl.) Gould: P27422s; P37099t

Dichanthelium linearifolium (Scribn.) Gould: P27527s

Dichanthelium oligosanthes (Schult.) Gould: P27423s; E30633a; E30659t; E31472b

Dichanthelium perlongum (Nash) Freckm.: E31936a

Dichanthelium villosissimum (Nash) Freckm.: P27414s; E30634a; E30658t; E31473b

Dichanthelium wilcoxianum (Vasey) Freckm.: S337s

Digitaria filiformis (L.) Koel.: P37243b

*Digitaria ischaemum (Screb.) Schreb.: E31510t

*Digitaria sanguinalis (L.) Scop.: P27917s; E31858b

*Echinochloa crus-galli (L.) P. Beauv.: P27980s

Echinochloa muricata (Michx.) Fern.: P28053s

*Eleusine indica (L.) Gaertn.: E31859b

Elymus canadensis L.: P27781s; E31511t

Elymus trachycaulus (Link) Gould: P27949s

Elymus virginicus L.: P27998s

*Elytrigia repens (L.) Desv.: P27947s; E31710a; INAIt

*Elytrigia smithii (Rydb.) Nevski: P27496s

*Eragrostis cilianensis (All.) Vign.: P28213s

Eragrostis hypnoides (Lam.) BSP: P28044s

*Eragrostis minor Host: P27778s

Eragrostis pectinacea (Michx.) Nees: P27836s

Eragrostis spectabilis (Pursh) Steud.: P27742s; P36231a; E31234t; E31955b

Eragrostis trichodes (Nutt.) Wood: P28284s; P37233b

*Festuca arundinacea Schreb.: P27492s

*Festuca pratensis Huds.: P27410s

Festuca subverticillata (Pers.) E.B. Alexeev: P27468s

*Festuca trachyphylla (Hack.) Krajina: P27554s 
Glyceria striata (Lam.) Hitchc.: P27482s

Heterostipa spartea (Trin.) Barkworth: P27412s; E30636a; E30660t; E31702b

Hordeum jubatum L.: P27548s

Koeleria macrantha (Ledeb.) Spreng.: P27421s; E30632a; E30602t; E31474b

Leersia oryzoides (L.) Swartz: P28043s

Leersia virginica Willd.: P28081s

Leptoloma cognatum (Schult.) Chase: P27916s; P36197a; E31512t; E31475b

*Lolium perenne L.: P27513s

Muhlenbergia mexicana (L.) Trin.: P28435s

Muhlenbergia racemosa (Michx.) BSP: P28277s

Muhlenbergia schreberi J.F. Gmel.: P28434s

Panicum capillare L. var. capillare: P28054s; E31513t; E31860b

Panicum virgatum L.: P27986s; E31269a; E31235t

Paspalum bushii Nash: P27859s; P36215a; E31514t; E31476b

Paspalum setaceum Michx. var. ciliatifolium (Michx.) Vasey: P28066s; P36232a; INAIt; E31861b

* Phalaris arundinacea L.: P27439s

*Poa bulbosa L.: P27324s

*Poa compressa L.: P27419s; E31711a; INAIt

Poa palustris L.: P27493s

*Poa pratensis L.: P27307s; E30635a; E30661t; E31666b

Schizachyrium scoparium (Michx.) Nash: P28425s; E31267a; E31236t; P37237b

*Setaria faberi R.A.W. Herrm.: P28069s; E31515t; E31863b

*Setaria glauca (L.) P. Beauv.: P28051s; P36213a; E31862b

*Setaria viridis (L.) P. Beauv.: P27725s

Sorghastrum nutans (L.) Nash: P28035s; P36206a; E31516t; P37246b

Spartina pectinata Link: P27997s; E31270a

Sphenopholis intermedia (Rydb.) Rydb.: P27486s

Sphenopholis obtusata (Michx.) Scribn.: P27514s

Sporobolus clandestinus (Biehler) Hitchc.: P28223s

Sporobolus compositus (Poir.) Merr.: P28418s; E31271a

Sporobolus cryptandrus (Torr.) Gray: P27511s; P36194a; P36160t; E31953b

Sporobolus heterolepis (Gray) Gray: INAIt

Sporobolus vaginiflorus (Torr.) A. Wood: P28212s; E31237t

Tridens flavus (L.) Hitchc.: P28090s; P37249b

Triplasis purpurea (Walt.) Chapm.: P28062s; P36230a; P366187t; P37250b

*Triticum aestivum L.: P27764s

Vulpia octoflora (Walt.) Rydb.: P27303s; P36708a; E30662t

\section{Pontederiaceae}

Zosterella dubia (Jacq.) Small: P28259.1s

\section{Potamogetonaceae}

*Potamogeton crispus L.: P28243s

Potamogeton nodosus Poir.: P27544s

Potamogeton pusillus L.: P28259.2s

Stuckenia pectinata (L.) Borner: P28258s

\section{Smilacaceae}

Smilax lasioneuron Hook.: P27813s

Smilax tamnoides L.: P27426s

\section{Sparganiaceae}

Sparganium eurycarpum Engelm.: P27984s 


\title{
Typhaceae
}

Typha latifolia L.: P28442s

\section{Zannichelliaceae}

Zannichellia palustris L.: P28590s

\section{DICOTS}

\section{Acanthaceae}

Ruellia humilis Nutt.: P27838s; E31822b

\section{Aceraceae}

Acer negundo L.: P27277s; P36709a; E31381t

Acer saccharinum L.: P27208s

Acer saccharum Marsh.: P28588s

\author{
Amaranthaceae \\ Amaranthus rudis J. Sauer: P28235s \\ *Amaranthus spinosus L.: P28430s \\ Amaranthus tuberculatus (Moq.) Sauer: P28432s \\ Froelichia floridana (Nutt.) Moq.: P27830s; P36210a; E31238t; E31823b \\ Froelichia gracilis (Hook.) Moq.: P27706s; E31937a; E31239t; E31446b
}

\author{
Anacardiaceae \\ Rhus aromatica Ait. var. arenaria (Greene) Fern.: P27320s; E31447b \\ Rhus aromatica Ait. var. aromatica: P27951s; E30613a; E30603t \\ Rhus glabra L.: P27334s; INAIa; P36190t; E31824b \\ Rhus hirta L.: P36223a; E31240t \\ Toxicodendron radicans (L.) Kuntze: P27719s; P36233a; E31241t; E31668b
}

\author{
Apiaceae \\ Cicuta maculata L.: P27993s \\ *Conium maculatum L.: P27768s \\ Cryptotaenia canadensis (L.) DC.: P27786s \\ *Daucus carota L.: P27726s; E31825b \\ Eryngium yuccifolium Michx.: INAIt \\ Heracleum maximum Bartr.: P27429s \\ Osmorhiza claytonii (Michx.) C.B. Clarke: P27436s \\ Osmorhiza longistylis (Torr.) DC.: P27435s \\ *Pastinaca sativa L.: P27791s \\ Sanicula canadensis L.: P27711s \\ Sanicula odorata (Raf.) Pryer \& Phillippe: P27469s \\ Spermolepis inermis (Nutt.) Math. \& Constance: P27739s; E31377a
}

\section{Apocynaceae}

Apocynum sibiricum Jacq.: P28086s; P36222a

\section{Araliaceae}

Aralia nudicaulis L.: P27845s 


\section{Asclepiadaceae}

Asclepias amplexicaulis Small: P27522s; E31359a; E31382t; E31826b

Asclepias hirtella (Pennell) Woodson: P36221a; P36166t

Asclepias incarnata L.: P27988s

Asclepias syriaca L. var. syriaca: P27704s; E31360a; E31383t; E31669b

Asclepias tuberosa L.: P27945s

Asclepias verticillata L.: P27946s; P36195a; E31384t; E31448b

Asclepias viridiflora Raf.: P27703s; E31361a; E31385t; E31449b

\section{Asteraceae}

*Achillea millefolium L.: P27507s; E30637a; E30663t; E31670b

Ageratina altissima (L.) R.M. King \& H. Rob.: P27966s

Ambrosia artemisiifolia L.: P28070s; P36209a; E31477t; E31827b

Ambrosia psilostachya DC.: Observed at s; E31275a; E31242t; E31828b

Ambrosia trifida L.: P28092s

Antennaria neglecta Greene: P27183s; E31312t

Antennaria plantaginifolia (L.) Hook.: P28118s; P36713a; E31647b

*Arctium lappa L.: P27954s

*Arctium minus Schk.: P28131s

Artemisia campestris L.: P28117s; E31478t

*Artemisia ludoviciana Nutt.: P28286s

Aster cordifolius L.: P28596s

Aster ericoides L.: P28252s; E31274a; E31243t

Aster lanceolatus Willd.: P28232s

Aster lateriflorus (L.) Britt.: P28448s

Aster oblongifolius Nutt.: P28283s

Aster ontarionis Wieg.: P28234s

Aster oolentangiensis Riddell: P28423s

Aster pilosus Willd.: Observed at s; E31273a; E31245t; E31956b

Aster prenanthoides Muhl.; P28271s

Aster puniceus L.: P28444s

Aster sericeus Vent.: P28214s; INAIa; E31244t

Bidens bipinnata $\mathrm{L} .: \mathrm{E} 31829 \mathrm{~b}$

Bidens cernua $\mathrm{L} .: \mathrm{P} 28229 \mathrm{~s}$

Bidens comosa (Gray) Wieg.: P28228s

Bidens vulgata Greene: P28123s

Brickellia eupatorioides (L.) Shinners: P28218s; P36224a; E31246t; P37234b

*Carduus nutans L.: P27541s; E31671b

*Centaurea biebersteinii DC.: P27846s

Chrysopsis camporum Greene: P27533s; E30638a; E30665t

*Cirsium arvense (L.) Scop.: P27777s

Cirsium discolor (Muhl.) Spreng.: P28050s; P36196a; E31479t

*Cirsium vulgare (Savi) Tenore: P27943s

Conyza canadensis (L.) Cronq.: P28036s; E31276a; E31247t; P37231b

Coreopsis palmata Nutt.: P27715s; P36207a; E31386t; E31451b

*Crepis tectorum L.: P27557s

Echinacea pallida (Nutt.) Nutt.: Observed at s; INAIt; E31453b

Eclipta prosrata (L.) L.: P28248s

Erechtites hieracifolia (L.) Raf.: P28225s

Erigeron annuus (L.) Pers.: P27497s; E31961a

Erigeron philadelphicus L.: P27432s

Erigeron strigosus Muhl.: P27520s; E31362a; E30664t; E31452b

Eupatoriadelphus purpureus (L.) R.M. King \& H. Rob.: P27989s

Eupatorium perfoliatum L.: P27990s 
Eupatorium serotinum Michx.: P27970s

Euthamia graminifolia (L.) Nutt.: E31480t

*Grindelia squarrosa (Pursh) Dunal: P28059s

Helenium autumnale L.: P28072s

*Helianthus annuus L.: P27720s

Helianthus hirsutus Raf.: P28603s

Helianthus mollis Lam.: P28061s

Helianthus occidentalis Riddell: P27924s; P36202a; E31481t; E31830b

Helianthus pauciflorus Nutt.: P28111s; E31250t; P37238b

*Helianthus petiolaris Nutt.: P36220a; P36172t; E31831b

Helianthus strumosus L.: E31454b

Helianthus tuberosus L.: P28107s

Heliopsis helianthoides (L.) Sweet: P27952s

Hieracium longipilum Torr.: P27935s; P36203a; E31482t

Ionactis linariifolius (L.) Greene: P28568s; E31272a; INAIt

Krigia virginica (L.) Willd.: P27177s; E31324a; E30604t; E31648b

Lactuca canadensis L.: P28445s; P36198a; E31483t; P37236b

Lactuca floridana (L.) Gaertn.: P28103s

*Lactuca serriola L.: P28064s; E31484t; E31832b

Liatris aspera Michx.: P28032s; E31278a; E31251t

* Matricaria discoidea DC.: P27779s

Oligoneuron rigidum (L.) Small: P28217s

Pseudognaphalium obtusifolium (L.) Hilliard \& Burtt: P28034s; E31277a; E31248t; E31951b

Ratibida pinnata (Vent.) Barnh.: P27987s

Rudbeckia hirta L.: P27730s; E31704a; E31672b

Rudbeckia laciniata L.: P28106s

Rudbeckia triloba L.: P28105s

Senecio plattensis Nutt.: P27164s; P36715a; E31649b

Silphium perfoliatum L.: P28099s

Solidago canadensis L.: P28077s; E31485t

Solidago juncea Ait.: INAIt

Solidago gigantea Ait.: P28080s

Solidago nemoralis Ait.: P27958s; E31279a; E31252t; P37235b

Solidago speciosa Nutt.: P28267s; INAIa; INAIt; E31455b

Solidago ulmifolia Muhl.: P28566s

*Taraxacum officinale Weber: P27312s

*Tragapogon dubius Scop.: P27411s; E30639a; E30666t; E31673b

Vernonia fasciculata Michx.: P28073s

\section{Balsaminaceae}

Impatiens capensis Meerb.: P28037s

Impatiens pallida Nutt.: P28005s

\section{Berberidaceae}

*Berberis thunbergii DC.: P28001s

Podophyllum peltatum L.: P27283s

\section{Betulaceae}

Betula nigra L.: P27161s

\section{Bignoniaceae}

*Catalpa speciosa Warder: P28121s; E31387t 


\section{Boraginaceae}

*Cynoglossum officinale L.: P27463s

*Echium vulgare L.: P27500s

Hackelia virginiana (L.) I.M. Johnston: P27919s

*Lappula squarrosa (Retz.) Dumort.: P27769s

Lithospermum croceum Fern.: P27163s; E30614a; E30605t; E31652b

Lithospermum incisum Lehm.: P27299s; INAIa; E31313t

\section{Brassicaceae}

*Alliaria petiolata (Bieb.) Cavara \& Grande: P27310s

*Alyssum alyssoides (L.) L.: P27289s

Arabis canadensis L.: P27465s

Arabis divaricarpa A. Nelson: P27556s

Arabis glabra (L.) Bernh.: P27343s; E30640a; E31314t

Arabis lyrata L.: P27154s; E30615a; E30606t; E31650b

*Barbarea vulgaris R. Br.: P27342s

*Berteroa incana (L.) DC.: P27449s

*Brassica nigra (L.) Koch: P27738s

*Capsella bursa-pastoris (L.) Medic.: P27279s

Cardamine bulbosa (Muhl.) BSP: P27331s

*Cardamine hirsuta L.: P27433s

Cardamine parviflora L.: P27309s

Cardamine pensylvanica Willd.: P28239s

Descurainia pinnata (Walt.) Britt.: P27165s; E30616a; E30667t; E31651b

*Draba nemorosa L.: P27284s

Draba reptans (Lam.) Fern.: P27156s; E31325a; E30668t

*Eriophila verna (L.) Chev.: P27191s; E31565t

*Erysimum cheiranthoides L.: E30670t

*Erysimum inconspicuum (S. Wats.) MacM.: P27416s

*Lepidium campestre (L.) R. Br.: P27407s

*Lepidium densiflorum Schrad.: P27409s; E31363a; E30669t; E31833b

Lepidium virginicum L.: P27302s; E30617a

Rorippa sessiliflora (Nutt.) A. Hitchc.: P27450s

*Rorippa sylvestris (L.) Besser: P27535s

* Sisymbrium altissimum L.: P27293s

*Thlaspi arvense L.: P27204s

\section{Cactaceae}

Opuntia fragilis (Nutt.) Haw.: P28065s

Opuntia macrorhiza Engelm.: P27862s; E31253t; E31445b

\section{Caesalpiniaceae}

Chamaecrista fasciculata (Michx.) Greene: P27934s; P36192a; E31487t; E31834b

Gleditsia triacanthos L.: P27417s; E31365a; E31488t; E31674b

Gymnocladus dioicus (L.) K. Koch: P27805s

\section{Campanulaceae}

Campanulastrum americanum (L.) Small: P27809s

Lobelia cardinalis L.: P27999s

Lobelia inflata L.: P28126s

Lobelia siphilitica L.: P28091s

Triodanis perfoliata (L.) Nieuwl.: P27505s; E30641a; E30671t; E31675b 


\section{Cannabinaceae}

*Cannabis sativa L.: P27833s; E31935a

*Humulus japonicus Sieb. \& Zucc.: P28285s

Humulus lupulus L.: P28124s

\section{Capparaceae}

Polanisia dodecandra (L.) DC.: P27737s; E31456b

Polanisia jamesii (Torr. \& Gray) Iltis: P27714s

\section{Caprifoliaceae}

*Lonicera morrowii Gray: P27295s; E31327a; P36182t

*Lonicera tatarica L.: P27281s; E31328a

*Lonicera xylosteum L.: E31676b

Sambucus canadensis L.: P27766s

Viburnum lentago L.: P27323s

*Viburnum opulus L.: P27428s

\section{Caryophyllaceae}

*Arenaria serpyllifolia L.: P27305s; E30644a; E31653b

*Cerastium brachypodum (Engelm.) B.L. Robins.: P27181s

*Cerastium fontanum Baum.: P27315s

*Dianthus armeria L.: P27775s

*Holosteum umbellatum L.: P27184s; E30618a; E31630b

*Myosoton aquaticum (L.) Moench.: P27442s

Paronychia canadensis (L.) Wood: P27831s

Paronychia fastigiata (Raf.) Fern.: P27921s

*Saponaria officinalis L.: P27707s; E31677b

Silene antirrhina L.: P27418s; E30643a; E30643t; E31679b

*Silene cserei Baumg.: P27418s; E30672t

*Silene dioica (L.) Clairv.: E31678b

Silene nivea (Nutt.) Otth.: P27787s

*Silene pratensis (Spreng.) Godron \& Gren: P27526s; E30642a

*Stellaria media (L.) Cyrillo: S227s

\section{Celastraceae}

Celastrus scandens L.: P27427s; E30645a; E31836b

Euonymus atropurpureus Jacq.: P28597s

\section{Ceratophyllaceae}

Ceratophyllum demersum L.: P28240s

\section{Chenopodiaceae}

*Chenopodium album L.: P36204a; E31388t; E31457b

*Chenopodium ambrosioides L.: P28048s

Chenopodium desiccatum A. Nels.: E31280a

Chenopodium pratericola Rydb.: P28049s

Chenopodium simplex (Torr.) Raf.: P27834s

Cycloloma atriplicifolium (Spreng.) Coult.: P27736s; E31939a; E31680b

*Salsola collina Pallas: P28115s; E31486t 


\section{Cistaceae}

Helianthemum bicknellii Fern.: P27851s; P36241a; P37241b

Helianthemum canadense (L.) Michx.: P27456s; E30646a; P36716t

Hudsonia tomentosa Nutt.: P27460s; E31326a

Lechea pulchella Raf.: P27867.2s

Lechea tenuifolia Michx.: P27868.1s; P37239b

\section{Convolvulaceae}

Calystegia sepium (L.) R. Br.: P27767s

*Convolvulus arvensis L.: P27852s

Stylisma pickeringii (Torr.) Gray: E31458b

\section{Cornaceae}

Cornus drummondii C.A. Mey.: P27976s; E31364a; E31681b

Cornus racemosa Lam.: P27490s

\section{Cucurbitaceae}

Sicyos angulatus L.: P28088s

\section{Cuscutaceae}

Cuscuta campestris Yuncker: P37100t

\section{Elaeagnaceae}

*Elaeagnus umbellata Thunb.: P27336s; P36714a; E31654b

\section{Euphorbiaceae}

Acalypha gracilens Gray: S142s

Acalypha rhomboidea Raf.: P27741s

Chamaesyce geyeri (Engelm.) Small: P27956s; P36211a; E31491t

Chamaesyce maculata (L.) Small: P28030s; P36174t; P37240b

Chamaesyce nutans (Lag.) Small: P28047s

*Chamaesyce prostrata (Ait.) Small: E31490t

Croton capitatus Michx.: S354s

Croton glandulosus L.: P27723s; P36218a; E31492t; E31837b

Crotonopsis linearis Michs.: INAIa; E31493t; E31838b

Euphorbia corollata L.: P27960s; E31366a; E31389t; E31459b

*Euphorbia esula L.: P27314s

Poinsettia dentata (Michx.) K1. \& Garcke: P28052s; P36193a; E31494t; E31839b

\section{Fabaceae}

Amorpha canescens Pursh: P27702s; E31367a; E31495t; E31460b

Amorpha fruticosa L.: P27817s; P36184t

Amphicarpaea bracteata (L.) Fern.: P28038s

Apios americana Medic.: P28102s

Baptisia alba (L.) Vent. E31390t

*Baptisia australis (L.) R. Br.: P37106a

Crotalaria sagittalis L.: P27962s

Dalea candida (Michx.) Willd.: S322s

Dalea purpurea Vent.: P27824s; E31368a; INAIt

Desmodium canadense (L.) DC.: P28104s

Desmodium glutinosum (Muhl.) A. Wood: P27842s

Desmodium illinoense Gray: P27914s; E31840b

*Kummerowia stipulacea (Maxim.) Makino: P28276s 
Lespedeza capitata Michx.: P28122s; E31281a; E31254t; E37232b

Lespedeza intermedia (S. Wats.) Britt.: P28567s

*Medicago lupulina L.: P27413s; E30647a; E31682b

*Melilotus albus Medic.: P27501s; E31369a; E31391t; E31461b

*Melilotus officinalis (L.) Pallas: P27406s; E31706a; E30674a; E31683b

*Robinia pseudoacacia L.: P27444s

*Securigera varia (L.) Lassen: P27524s

Strophostyles helvula (L.) Ell. var. helvula: P27933s; P36243a; E31841b

Strophostyles helvula (L.) Ell. var. missouriensis (S. Wats.) Britt.: P27765s

Strophostyles leiosperma (Torr. \& Gray) Piper: P27925s

Tephrosia virginiana (L.) Pers.: P27728s; E31282a; E31255t; E31462b

*Trifolium arvense L.: P27747s

*Trifolium campestre Schreb.: P27733s

*Trifolium hybridum L.: P28455s

*Trifolium pratense L.: P27473s; E31842b

*Trifolium repens L.: P27474s

*Vicia villosa Roth: P27476s

\section{Fagaceae}

Quercus alba L.: P28443s

Quercus x bushii Sarg.: E31634b

Quercus macrocarpa Michx.: P27319s

Quercus marilandica Muench.: E31655b

Quercus muhlenbergii Englem.: P27475s

Quercus palustris Muench.: P28094s

Quercus rubra L.: P28042s

Quercus velutina Lam.: P27187s; E30619a; E31256t; E31633b

\section{Fumariaceae}

Corydalis micrantha (Engelm.) Gray: P27287s

Dicentra cucullaria (L.) Bernh.: P27203s

\section{Gentianaceae}

Gentiana puberulenta J. Pringle: INAIt

\section{Geraniaceae}

Geranium carolinianum L.: P27553s; E31684b

Geranium maculatum L.: P27329s

\section{Grossulariaceae}

Ribes missouriense Nutt.: P27189s; P36227a; P36181t; E31636b

\section{Haloragidaceae}

*Myriophyllum spicatum L.: P28260s

\section{Hydrophyllaceae}

Ellisia nyctelea L.: P27278s

Hydrophyllum virginianum L.: P27338s

\section{Hypericaceae}

*Hypericum perforatum L.: P27700s

Hypericum punctatum Lam.: P27996s

Hypericum sphaerocarpum Michx.: P27797s 


\section{Juglandaceae}

Carya cordiformis (Wangenh.) K. Koch: P27466s

Carya ovata (Mill.) K. Koch: P28119s

Carya tomentosa (Poir.) Nutt.: P28130s

Juglans cinerea L.: P28584s

Juglans nigra L.: P27291s

\section{Lamiaceae}

Agastache nepetoides (L.) Ktze.: P27944s

Hedeoma hispida Pursh: P27415s

*Leonurus cardiaca L.: P27708s; E31685b

Lycopus americanus Muhl.: P27955s

Lycopus uniflorus Michx.: P28079s

* Mentha arvensis L.: P27992s

Monarda fistulosa L. var. fistulosa: P27841s; E31843b

Monarda fistulosa L. var. mollis (L.) Benth.: P27950s

Monarda punctata L.: P27931s; E31283a; E31257t; E31844b

*Nepeta cataria L.: P27776s; P36164t; E31845b

Physostegia virginiana (L.) Benth.: P28078s

*Prunella vulgaris L.: P27967s

Pycnanthemum virginianum (L.) Dur. \& B.D. Jacks.: P28098s

Salvia azurea Michx. \& Lam.: P28060s

Scutellaria lateriflora L.: P28075s

Scutellaria leonardii Epling: P27837s

Scutellaria ovata Hill: P27744s

Stachys hispida Pursh: P28247s

Stachys tenuifolia Willd.: P27799s

Teucrium canadense L.: P27801s

Trichostema dichotomum L.: P27941s

\section{Linaceae}

Linum sulcatum Riddell: P27823s

\section{Lythraceae}

Ammannia coccinea Rottb.: P28237s

*Lythrum salicaria L.: P28249s

Rotala ramosior (L.) Koehne: P27866s

\section{Malvaceae}

Callirhoe triangulata (Leavenw.) Gray: P27762s; P36201a; E31496t; E31686b

Hibiscus laevis All.: P28075s

*Malva neglecta Wallr.: P28251s

\section{Menispermaceae}

Menispermum canadense L.: P27484s

\section{Molluginaceae}

*Mollugo verticillata L.: P27705s; P36219a; E31258t; E31687b

\section{Moraceae}

*Morus alba L.: P27285s; P36225a; E31259t

*Morus tatarica L.: E31688b 


\section{Nelumbonaceae}

Nelumbo lutea (Willd.) Pers.: P28003s

\section{Nyctaginaceae}

Mirabilis hirsuta (Pursh) MacM.: P27793s

*Mirabilis nyctaginea (Michx.) MacM.: P27521s; E31370a; E31497t; E31689b

\section{Nymphaeaceae}

Nymphaea tuberosa Paine: P28263s

\section{Oleaceae}

Fraxinus lanceolata Borkh.: P27489s

Fraxinus pennsylvanica Marsh.: P36238a; P36165t

*Syringa vulgaris L.: P27321s

\section{Onagraceae}

Circaea lutetiana L.: P27709s

Epilobium ciliatum Raf.: P28438s

Gaura longiflora Spach: P28050s

Oenothera biennis L.: P28083s: P37102a

Oenothera clelandii W. Dietr., Raven, \& W.L. Wagner: P27729s; P36205a; E31498t; E31463b

Oenothera laciniata Hill: P27926s

\section{Orobanchaceae}

Orobanche fasciculata Nutt.: G2634s

\section{Oxalidaceae}

Oxalis stricta L.: P27525s; E30648a

Oxalis violacea L.: E31637b

\section{Papaveraceae}

Sanguinaria canadensis L.: P27193s

\section{Phrymaceae}

Phryma leptostachya L.: P27844s

\section{Phytolaccaceae}

Phytolacca americana L.: S110s; E31846b

\section{Plantaginaceae}

Plantago aristata Michx.: P27758s

*Plantago lanceolata L.: P27735s

Plantago patagonica Jacq.: P27508s; E30649a; E30675t; E31690b

Plantago rugelii Decne.: P27840s

Plantago virginica L.: P27448s

\section{Polemoniaceae}

Phlox divaricata $\mathrm{L} .: \mathrm{P} 27194 \mathrm{~s}$

\section{Polygalaceae}

Polygala polygama Walt.: P27516s; E31285a; E30676t; E31464b

Polygala sanguinea L.: Observed at a.

Polygala verticillata L.: P22291s 


\section{Polygonaceae}

Antenoron virginianum (L.) Roberty \& Vautier: P28039s

*Fallopia convolvulus (L.) A. Love: P27835s; E31691b

Fallopia scandens (L.) Holub.: P28128s; E31499t

Persicaria amphibium (L.) S.F. Gray: P28230s

*Persicaria cespitosa (Blume) Nakai: P28068s

*Persicaria hydropiper (L.) Opiz: P28093s

Persicaria lapathifolia (L.) S.F. Gray: P28057s

Persicaria pensylvanica (L.) Small: P27978s

Persicaria punctata (Ell.) Small: P28056s

*Persicaria vulgaris Webb \& Moq.: P27979s

Polygonella articulata (L.) Meisn.: P28220s; E31284a; E31260t

*Polygonum aviculare L.: E31392t

Polygonum ramosissimum Michx.: P28215s

Polygonum tenue Michx.: P27930s; P36234a; P36171t; P37244b

*Rumex acetosella L.: P27313s; E30650a; E30607t; E31656b

Rumex altissimus Wood: P27540s

*Rumex crispus L.: P27546s

Rumex verticillatus L.: P27816s

\section{Portulacaceae}

Claytonia virginica L.: P27199s

*Portulaca oleracea L.: P27865s

Talinum rugospermum Holz.: P27740s; P36242a; P22637t; E31847b

\section{Primulaceae}

Androsace occidentalis Pursh: P27157s; E31329a; E31315t; E31657b

Lysimachia ciliata L.: P27798s

\section{Ranunculaceae}

Anemone canadensis L.: P27317s

Anemone caroliniana Walt.: P27155s; INAIa

Anemone cylindrica Gray: P27538s; INAIa

Anemone quinquefolia L.: P27202s

Anemone virginiana L.: P27790s

Aquilegia canadensis L.: P27286s

Caltha palustris L.: P27316s

Clematis virginiana L.: P28273s

Ranunculus abortivus L.: P27168s; E30677t

Ranunculus fascicularis Bigel.: P27301s

Ranunculus pensylvanicus L.f.: P27972s

Ranunculus septentrionalis Poir.: P27330s

Thalictrum dasycarpum Fisch. \& Lall.: P27802s

\section{Rhamnaceae}

Ceanothus americanus L.: P27731s; P36199a; INAIt

Ceanothus herbaceus Raf.: P27458s

*Rhamnus cathartica L.: P27328s

\section{Rosaceae}

Agrimonia gryposepala Wallr.: P27965s

Agrimonia pubescens Wallr.: P28002s

Crataegus calpodendron (Ehrh.) Medic.: P27985s 
Fragaria virginiana Duchesne: P27294s

Geum canadense Jacq.: P27710s; E31500t

Geum triflorum Pursh: P27153s

Malus coronaria (L.) Mill.: P27174s

Malus ioensis (Wood) Britt.: P27276s

*Malus pumila Mill.: P27205s

Physocarpus opulifolius (L.) Maxim.: P28004s

*Potentilla argentea L.: P27297s

Potentilla arguta Pursh: INAIt

*Potentilla inclinata Vill.: P27405s

Potentilla norvegica L.: P27754s

*Potentilla recta L.: P27504s; E31371a; E30678t; E31692b

Potentilla simplex Michx.: P28602s; INAIt

Prunus americana Marsh.: P27162s

Prunus nigra Ait.: P27160s

Prunus serotina Ehrh.: P27341s; E30620a; E31318t; E31658b

Prunus susquehanae Willd.: INAIa; E31316t

Prunus virginiana L.: P28296s; P36229a; E31317t

Rosa carolina L.: P27506s; P37105a; E31393t

*Rosa multiflora Thunb.: P27545s; P36712a

Rosa suffulta Greene: P28100s

Rubus allegheniensis Porter: P27536s

Rubus argutus Link: P27477s

Rubus baileyanus Britt.: E31394t

Rubus flagellaris Willd.: P27445s

Rubus occidentalis L.: P27461s; P36228a; P36163t; E31693b

Rubus pensilvanicus Poir.: E31848b

\section{Rubiaceae}

Cephalanthus occidentalis L.: P27807s

Diodia teres Walt.: P27937s; P36239a; E31261t; E31849b

Galium aparine L.: P27288s; E30679t; E31694b

Galium circaezans Michx.: P28579s

Galium concinnum Torr. \& Gray: P27843s

Galium triflorum Michx.: P27743s

\section{Rutaceae}

Ptelea trifoliata L.: P27750s; INAIa; E30680t

Zanthoxylum americanum Mill.: P27173s; E31372a

\section{Salicaceae}

Populus deltoides Marsh.: P27340s; P36711a

Populus grandidentata Michx.: P27333s

Populus tremuloides Michx.: P27961s

Salix amygdaloides Anderss.: P27804s

Salix interior Rowlee: P27206s

Salix nigra Marsh.: P27318s

\section{Santalaceae}

Comandra umbellata (L.) Nutt.: P27335s; INAIt

\section{Saxifragaceae}

Penthorum sedoides L.: P27973s 


\section{Scrophulariaceae}

Agalinis tenuifolia (Vahl) Raf.: P28451s

Aureolaria grandiflora (Benth.) Pennell: S216s

Bacopa rotundifolia (Michx.) Wettst.: P27971s

Besseya bullii (Eat.) Rydb.: P27539s; E31659b

*Chaenorrhinum minus (L.) Lange: P 27854s

Gratiola neglecta Torr.: P27549s

*Linaria vulgaris Mill.: P27795s

Lindernia dubia (L.) Pennell var. dubia: P29046s

Mimulus ringens L.: P28089s

Nuttallanthus canadensis (L.) D. Sutton: P27178s; E30621a; E30608t; E31660b

Penstemon grandiflorus Nutt.: P36188t; P37247b

Penstemon pallidus Small: P27325s; E30651a; E30609t; E31695b

Scrophularia lanceolata Pursh: P27446s

*Verbascum blattaria L.: P27953s

*Verbascum thapsus L.: P27995s; E31707a; E31501t; E31850b

*Veronica arvensis L.: P27298s; E30622a; E30682t; E31661b

*Veronica dillenii Crantz: P27180s

Veronica peregrina L.: P27282s

Veronicastrum virginicum (L.) Farw.: INAIt

\section{Solanaceae}

Physalis heterophylla Nees: P27515s; E31373a; E31502t; E31851b

Physalis subglabrata Mack. \& Bush: P28236s; E31465b

Physalis virginiana Mill.: P27459s; E31374a; P36186t; E31696b

Solanum carolinense L.: P27724s; P37101t; E31697b

*Solanum dulcamara L.: P36237a; E30681t

Solanum ptychanthum Dunal: P27745s; E31503t

*Solanum rostratum Dunal: P28031s

\section{Tiliaceae}

Tilia americana L.: P27443s

\section{Ulmaceae}

Celtis occidentalis L.: P27172s; P37103a; E31505t

Ulmus americana L.: P27167s; P36200a

*Ulmus pumila L.: P27152s; E31504t; E31698b

Ulmus rubra Muhl.: P27196s

\section{Urticaceae}

Boehmeria cylindrica (L.) Sw.: P28007s

Laportea canadensis (L.) Wedd.: P28082s

Parietaria pensylvanica Muhl.: P27455s; E31852b

Pilea pumila (L.) Gray: P28272s

Urtica gracilis Ait.: P27748s

\section{Verbenaceae}

Phyla lanceolata (Michx.) Greene: P27839s

Verbena bracteata Lag. \& Rodr.: P27734s; E31375a

Verbena hastata L.: P28055s

Verbena stricta Vent.: P27869s; E31286a; E31262t; E31466b

Verbena urticifolia L.: P27808s 


\section{Violaceae}

Viola pedata L.: P27186s; E30623a; E31319t; E31662b

Viola pratincola Greene: P27327s; P37097t

Viola pubescens Ait.: P27339s

*Viola rafinesquii Greene: P27322s; E30610t; E31663b

Viola sororia Willd.: P27185s

\section{Vitaceae}

Parthenocissus inserta (Kern.) K. Fritsch: P27811s; E31853b

Parthenocissus quinquefolia (L.) Planch.: P27939s; P36226a; E31506t

Vitis riparia Michx.: P27447s; P36236a; E31395t; E31854b 

Illinois Natural History Survey

I-Building

1816 South Oak Street

Champaign, Illinois 61820

217-333-6880

A Division of the Illinois Department of Natural Resources

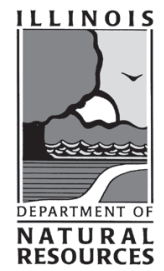

